\title{
On the Throughput Capacity of Heterogeneous Wireless Networks
}

\author{
Pan Li, Member, IEEE, and Yuguang Fang, Fellow, IEEE
}

\begin{abstract}
A substantial body of the literature exists addressing the capacity of wireless networks. However, it is commonly assumed that all nodes in the network are identical. The issue of heterogeneity has not been embraced into the discussions. In this paper, we investigate the throughput capacity of heterogeneous wireless networks with general network settings. Specifically, we consider an extended network with $n$ normal nodes and $m=n^{b}(0 \leq b \leq 1)$ more powerful helping nodes in a rectangular area with width $s(n)$ and length $n / s(n)$, where $s(n)=n^{w}$ and $0 \leq w \leq 1 / 2$. We assume that there are $n$ flows in the network. All the $n$ normal nodes are sources while only randomly chosen $n^{d}(0 \leq d \leq 1)$ normal nodes are destinations. We further assume that the $n$ normal nodes are uniformly and independently distributed, while the $m$ helping nodes are either regularly placed or uniformly and independently distributed, resulting in two different kinds of networks called Regular Heterogeneous Wireless Networks and Random Heterogeneous Wireless Networks, respectively. We show that network capacity is determined by the shape of the network area, the number of destination nodes, the number of helping nodes, and the bandwidth of helping nodes. We also find that heterogeneous wireless networks can provide throughput higher in the order sense than traditional homogeneous wireless networks only under certain conditions.
\end{abstract}

Index Terms-Heterogeneous wireless networks, extended networks, achievable throughput

\section{INTRODUCTION}

$\mathrm{T}$ O date, a tremendous amount of effort has been made on the capacity of wireless networks. Gupta and Kumar [14] are the first to show that the per-node throughput capacity is $\Theta(1 / \sqrt{n \log n})$ bits per second in random ad hoc networks, and the per-node transport capacity is $\Theta(1 / \sqrt{n})$ bit-meters per second in arbitrary ad hoc networks, respectively, where $n$ is the number of nodes in the network. Following [14], extensive research on capacity has been conducted in both static ad hoc networks, such as [6], [9], [10], [11], [36], and mobile ad hoc networks, such as [5], [12], [13], [22], [28], [34], [41], [42], considering the use of omnidirectional antennas. When directional antennas are employed, [37] and [26] study the capacity, and [27] explores the connectivity, of the networks, respectively.

However, all the works above investigate homogeneous wireless networks where all nodes are assumed identical. The issue of heterogeneity has not been embraced into the discussions. But in practice, there are many applications in which wireless networks are heterogeneous. For example, in a city-wide wireless network, those wireless devices mounted on top of the buildings are usually much more powerful than normal network users. To give another example, in a wireless network deployed in battlefields, many military vehicles like tanks and planes are much more powerful than normal soldiers, and the communications

- P. Li is with the Department of Electrical and Computer Engineering, Mississippi State University, 229 Simrall Engineering Bulding, Mississippi State, MS 39762-9571. E-mail: li@ece.msstate.edu.

- Y. Fang is with the Department of Electrical and Computer Engineering, University of Florida, NEB 435, Gainesville, FL 32611.

E-mail: fang@ece.ufl.edu.

Manuscript received 4 Aug. 2010; revised 18 Sept 2011; accepted 6 Oct. 2011; published online 3 Nov. 2011.

For information on obtaining reprints of this article, please send e-mail to: tmc@computer.org, and reference IEEECS Log Number TMC-2010-08-0368. Digital Object Identifier no. 10.1109/TMC.2011.239. between them may even be carried out at a higher frequency and have much larger bandwidth.

Some researchers propose to connect the more powerful nodes with a wired network, resulting in the so-called "hybrid wireless networks" [1], [18], [20], [24], [29], [46], [47], [48]. However, this approach has some shortcomings. First, it is cost-expensive since the powerful nodes they use are in fact base stations and an optical network connecting the base stations needs to be established first. Second, it is timeconsuming to set up such a backbone optical network. Third, current results show that hybrid wireless networks can achieve higher throughput than traditional static homogeneous ad hoc networks only when the number of base stations is $\Omega(\sqrt{n})$ [29], which makes this approach even more expensive. Moreover, in some cases base stations and a wired backbone network are even unavailable. For example, in the rescue affairs after natural disasters like earthquakes, the existing infrastructures may have been damaged and we need to set up a network without them immediately. In such cases, a pure wireless network would be more helpful and of much more interest.

Thus, herein we consider heterogeneous wireless networks, in which all the transmissions are carried out via wireless medium, and normal nodes and some more powerful helping nodes coexist. In heterogeneous wireless networks, [23], [25], [35], [49] propose medium access control (MAC) protocols, [3], [31], [32], [33], [44], [45] develop routing algorithms, and [19] studies topology control, respectively. Jain et al. [15] and Sollacher [39] also look into the impact of interference on the performance of ad hoc networks. However, the asymptotic capacity problem has not been well studied yet.

In this paper, we investigate the throughput capacity of heterogeneous wireless networks. We aim to find out that instead of placing base stations interconnected by a wired 


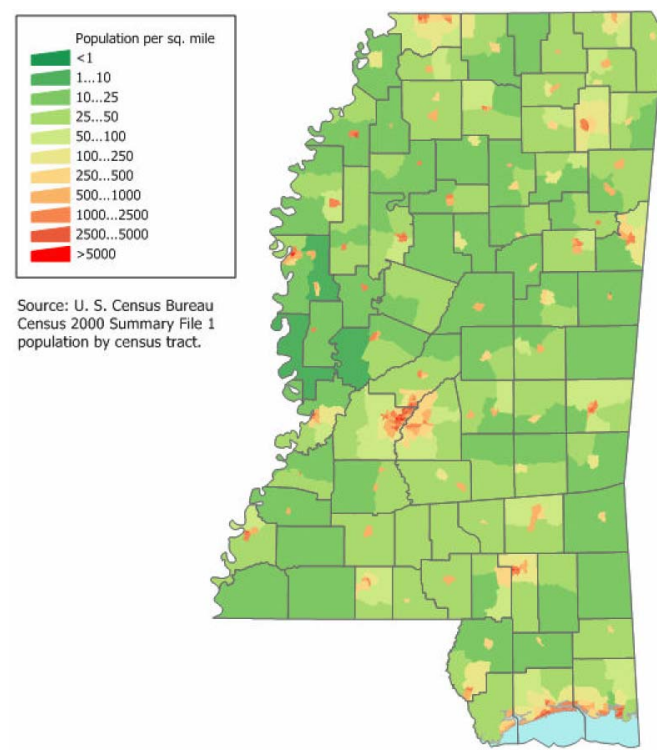

Fig. 1. The population distribution in Mississippi obtained from Wikipedia.

network, how we can improve the capacity of homogeneous ad hoc networks by deploying some more powerful wireless helping nodes. Notice that most of the previous research on capacity assumes the network area is a square, and the traffic is symmetric implying that the number of source nodes is the same as the number of destination nodes. However, those are only special cases. In practice, the shape of a network area is determined by the distribution of network users, which is further dependent on many factors such as geographical characteristics. For example, about 75 percent of the population of Utah lies in a corridor which stretches approximately from Brigham City at the north end to Nephi at the south end along the Wasatch Mountains [43]. Besides, as shown in Fig. 1, the population of Mississippi is approximately distributed in a rectangle, too. So, instead of a square, we may consider the network area in rectangle. Besides, in many applications such as peer-to-peer (P2P) networking, the number of source nodes is usually different from that of destination nodes. Thus, instead of symmetric traffic, we consider asymmetric traffic. In the literature, Liu et al. [30] study the capacity of 2D strip hybrid wireless networks with symmetric traffic. Toumpis [40] studies the throughput capacity of 2D square ad hoc networks with asymmetric traffic. Li and Fang [20] investigate the impacts of both network area shape and traffic pattern on the throughput capacity of hybrid wireless networks. But, when it comes to heterogeneous wireless networks, the situation becomes more complicated and it is worthwhile to seriously analyze the capacity of the networks again.

Specifically, in this paper, we consider an extended network with $n$ normal nodes and $m=n^{b}(0 \leq b \leq 1)$ more powerful helping nodes in a rectangular area with width $s(n)$ and length $n / s(n)$, where $s(n)=n^{w}$ and $0 \leq w \leq 1 / 2$. We consider that there are $n$ flows in the network. All the $n$ normal nodes are sources while only $n^{d}(0 \leq d \leq 1)$ randomly chosen normal nodes are destinations. Helping nodes do not serve as data sources or data destinations.
Instead, they only help relay packets for the normal nodes. Moreover, notice that in real wireless networks, the normal nodes are usually WiFi users which have low data rates while the helping nodes may use more advanced technologies such as cognitive radio, Multi-Input and Multi-Output (MIMO), and Ultra-WideBand (UWB), which can provide much higher data rates. Without loss of generality, we assume the normal users share a bandwidth of 1 and the helping nodes have much higher bandwidth for the transmissions between themselves. We further assume that the $n$ normal nodes are uniformly and independently distributed, while the $m$ helping nodes are either regularly placed or uniformly and independently distributed, resulting in two different kinds of networks called Regular Heterogeneous Wireless Networks and Random Heterogeneous Wireless Networks, respectively. We attempt to find out what a heterogeneous wireless network with general network settings can do by deriving a lower bound on the throughput capacity. Our results demonstrate that network capacity is determined by the shape of the network area, the number of the destination nodes, the number of the helping nodes, and the bandwidth of the helping nodes. We also explore the conditions under which heterogeneous wireless networks can provide higher throughput than traditional pure static ad hoc wireless networks. We discover that the bandwidth of the helping nodes should be $\omega\left(n^{w}\right)$ when $w+b / 2-1 / 2 \leq$ 0 and $w-\min \{b, d\}<0$, and $\omega\left(n^{\frac{1-b}{2}}\right)$ when $w+b / 2-1 / 2>$ 0 and $w-\min \{b, d\}<0$, respectively. Furthermore, we notice that increasing helping nodes' bandwidth does not necessarily always lead to an increase in network capacity. We find that the bandwidth of the helping nodes should be upper bounded by $\min \left\{n^{b}, n^{d}\right\}$ when $w+b / 2-1 / 2 \leq 0$, and by $\min \left\{n^{\frac{b}{2}-w+\frac{1}{2}}, n^{d-\frac{b}{2}-w+\frac{1}{2}}\right\}$ when $w+b / 2-1 / 2>0$, respectively. More bandwidth than that cannot increase the network capacity further since the other factors will be the bottleneck of the network.

The rest of this paper is organized as follows: Section 2 gives the notations, definitions, and models including topology model, traffic model, and achievable transmission rate model, that we use throughout this paper. In Sections 3 and 4, we derive an achievable throughput of heterogeneous wireless networks, when helping nodes are regularly and randomly distributed, respectively. Some extreme cases are discussed in Section 5. More insights into the results are given in Section 6. We finally conclude this paper in Section 7.

\section{Notations, Definitions, And Models}

Here, we introduce some notations, definitions, and models that will be used in this paper.

\subsection{Notations}

We use the following notations [17]:

- $f(n)=O(g(n))$ means $f(n)$ is asymptotically upper bounded by $g(n)$, i.e., $\lim \sup _{n \rightarrow \infty}\left|\frac{f(n)}{g(n)}\right|<\infty$.

- $f(n)=\Omega(g(n))$ means $f(n)$ is asymptotically lower bounded by $g(n)$, i.e., $\liminf _{n \rightarrow \infty}\left|\frac{f(n)}{g(n)}\right|>0$. 
- $f(n)=\Theta(g(n))$ means $f(n)$ is asymptotically tight bounded by $g(n)$, i.e., $0<\liminf _{n \rightarrow \infty}\left|\frac{f(n)}{g(n)}\right| \leq$ $\limsup _{n \rightarrow \infty}\left|\frac{f(n)}{g(n)}\right|<\infty$.

- $\quad f(n)=o(g(n))$ means $f(n)$ is asymptotically negligible with respect to $g(n)$, i.e., $\lim _{n \rightarrow \infty}\left|\frac{f(n)}{g(n)}\right|=0$.

- $\quad f(n)=\omega(g(n))$ means $f(n)$ is asymptotically dominant with respect to $g(n)$, i.e., $\lim _{n \rightarrow \infty}\left|\frac{f(n)}{g(n)}\right|=\infty$.

\subsection{Definitions}

Throughput. As defined in the usual way, the time average of the number of bits per second that can be transmitted by each node to its destination is called the per-node throughput.

Achievable throughput. Let $\lambda_{i}(n)$ denote the throughput of node $i$. We say that a per-node throughput, denoted by $\lambda(n)$, is achievable by all nodes if there exists a spatial and temporal scheduling scheme such that $\lambda_{i}(n) \geq$ $\lambda(n)$ for all $i \in[1, n]$, and is achievable on average if there exists a spatial and temporal scheduling scheme such that $\frac{1}{n} \sum_{i=1}^{n} \lambda_{i}(n) \geq \lambda(n)$. In this paper, we will derive a pernode throughput achievable by all nodes. Notice that a throughput achievable by all nodes is also achievable on average.

Throughput capacity. We say that the throughput capacity of a network is lower bounded by $\Omega(f(n))$ bits per second if there is a deterministic constant $c>0$ such that [14]

$$
\lim _{n \rightarrow+\infty} \operatorname{Prob}(\lambda(n)=c f(n) \text { is feasible })=1 .
$$

\subsection{Network Model}

We consider an extended network with $n$ normal nodes and $m=n^{b} \quad(0 \leq b \leq 1)$ more powerful helping nodes in a rectangular area with width $s(n)$ and length $n / s(n)$, where $s(n)=n^{w}$ and $0 \leq w \leq \frac{1}{2}$. We assume the $n$ normal nodes are uniformly and independently distributed, while the $m$ helping nodes are either regularly placed or uniformly and independently distributed. The resulting two kinds of networks are called Regular Heterogeneous Wireless Networks and Random Heterogeneous Wireless Networks, respectively, which will be discussed in Sections 3 and 4 shortly.

As shown in Fig. 2, the proposed network model has a two-tier hierarchy and all the transmissions in the network are carried out via wireless medium.

\subsection{Traffic Model}

Instead of symmetric traffic mostly assumed in the literature, we assume the network has asymmetric traffic. Specifically, we consider there are $n$ flows in the network. All the $n$ normal nodes are sources while only randomly chosen $n^{d}(0 \leq d \leq 1)$ normal nodes are destinations. When $d=1$, we follow the process in [13] to choose random sender-receiver pairs so that each node is a source node for one flow and a destination node for at most $\mathrm{O}(1)$ flows. Helping nodes do not serve as data sources or data destinations. Instead, they only help relay packets for the normal nodes.

\subsection{Achievable Transmission Rate}

Let $d_{i j}$ denote the distance between a node $i$ and another node $j$. The reception power at node $j$ of the signal from

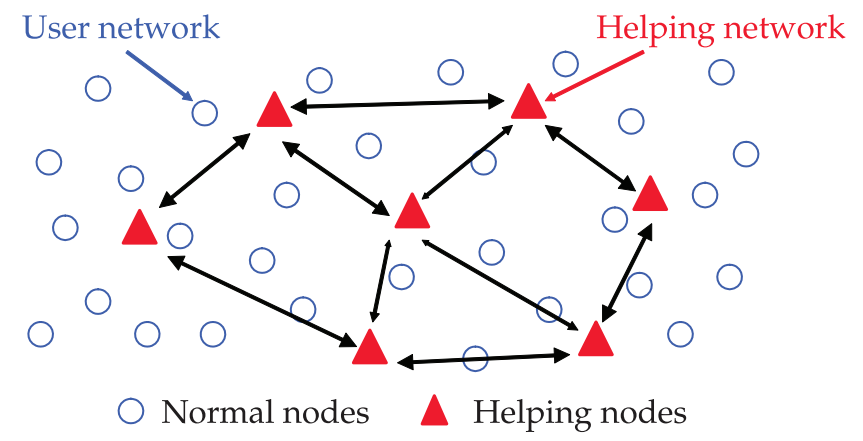

Fig. 2. Our proposed two-tier network model.

node $i$, denoted by $P_{i j}$, follows the power propagation model described in [2], [4], [8], and [10], i.e.,

$$
P_{i j}=C P_{i} /\left(1+d_{i j}\right)^{\gamma},
$$

where $P_{i}$ is the transmission power of node $i, \gamma$ is the path loss exponent, and $C$ is a constant related to the antenna profiles of the transmitter and the receiver, wavelength, and so on. As a common assumption, we assume $\gamma>2$ in outdoor environments [38].

We consider the Shannon Capacity as the achievable transmission rate between two nodes. Specifically, a transmission from node $i$ to node $j$ can achieve transmission rate, $R_{i j}$, which is calculated as follows:

$$
R_{i j}=W \log \left(1+S I N R_{i j}\right),
$$

where $W$ is the channel bandwidth, and

$$
S I N R_{i j}=\frac{C P_{i} /\left(1+d_{i j}\right)^{\gamma}}{N+\sum_{k \neq i} C P_{k} /\left(1+d_{i j}\right)^{\gamma}}
$$

is the Signal-to-Interference plus Noise Ratio (SINR) of the signal from node $i$ to node $j$. In this paper, we assume the $n$ normal nodes employ the same transmission power $P(n)$ for all their transmissions, and the $m$ helping nodes use the same transmission power $P^{\prime}(m)$ for the transmissions among themselves.

\section{An Achievable Throughrut of Regular Heterogeneous Wireless Networks}

In this section, we derive an achievable per-node throughput of regular heterogeneous wireless networks when $0<w \leq \frac{1}{2}, 0<b<1$, and $0<d<1$. We assume the helping nodes are regularly placed. As shown in Fig. 3, transmissions in the network can be carried out either in user mode or in help mode. Specifically, in user mode, packets are forwarded from a source node to a destination node with the help of only normal nodes, i.e., without the help of helping nodes. In help mode, packets are first transmitted from a source node to the helping network, and then forwarded to the intended destination user. Besides, we assume all the normal nodes have a total bandwidth of 1 , which is split into three frequency bands, i.e., $W_{1}$ for ad hoc transmissions in user mode, $W_{2}$ for uplink transmissions in help mode, and $W_{3}$ for downlink transmissions in help mode, respectively. Thus, 


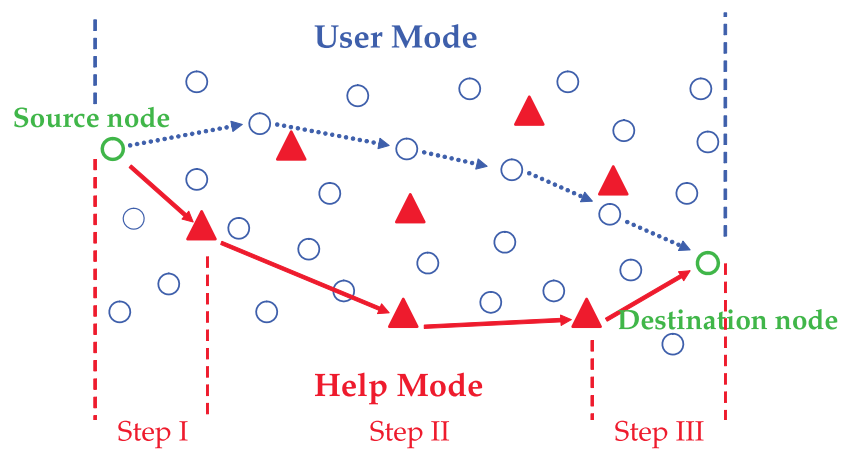

Fig. 3. Transmissions in two modes in the network.

$$
W_{1}+W_{2}+W_{3}=1 .
$$

Assume the ad hoc transmissions in help mode have a bandwidth $W_{4}$, which may have much higher order than 1 since it is the bandwidth of backbone helping network. We also assume that helping nodes have two network interfaces so that they can work on their own frequency band and the normal nodes' frequency band at the same time without any mutual interference. Note that normal nodes and helping nodes communicating on normal nodes' frequency band only need one antenna. We will make schedules so that transmissions on different subbands take place at different times (mini-slots) and will not interfere with each other.

Let $T_{u}$ and $T_{h}$ denote an achievable per-node throughput by all nodes when all the transmissions are carried out in user mode $\left(W_{1}=1\right)$ and in help mode $\left(W_{2}+W_{3}=1\right)$, respectively. Then, a per-node throughput achievable by all nodes in heterogeneous wireless networks, denoted by $T$, can be calculated as follows:

$$
T=\max \left\{T_{u}, T_{h}\right\} .
$$

In the following, we will derive the throughput in user mode and in help mode, respectively. The basic idea is that given any source-destination pair, if we could find a path between them and the scheduling for all nodes on the path to transmit, then the resulting throughput will be an achievable throughput in this network. Thus, in order to find an achievable throughput in the network, we need to give the scheduling and routing strategies first.

\subsection{Achievable Throughput in User Mode}

We first introduce the scheduling strategy. We divide the network into squares with length $l=\sqrt{c_{1} \log n}$, where $c_{1}\left(c_{1}>1\right)$ is a constant. Then, we have the following lemma [21].

Lemma 1. Every square contains at least one normal node with high probability (w.h.p.).

Besides, in the network, we allow a transmission between two normal nodes only when they are located in two neighboring squares. Notice that each square can have at most four neighboring squares. Thus, we arrive at the following lemma.

Lemma 2. Each square in the network can transmit at a transmission rate $c_{2} W_{1}$ where $c_{2}$ is a deterministic positive constant.

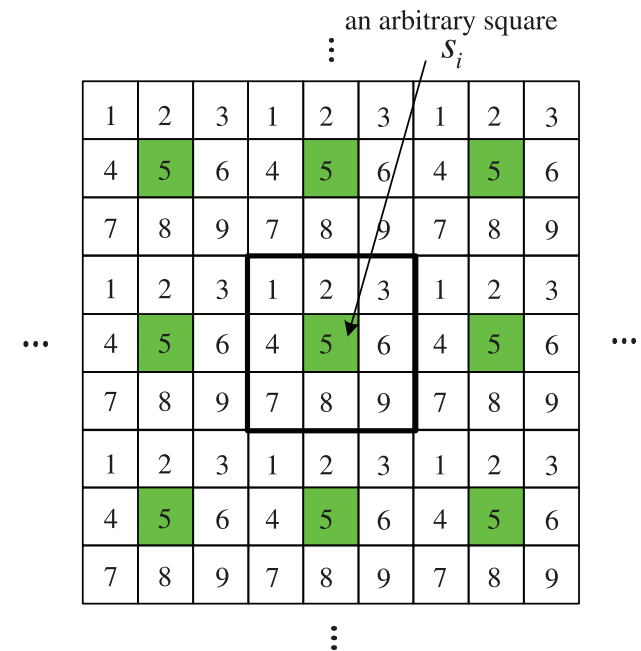

Fig. 4. An example for dividing the network into groups of nine squares.

Proof. We further divide the network into groups each of which contains nine squares. As shown in Fig. 4, the nine squares in each group are numbered from 1 to 9 in the same way. We also divide time into sequences of successive slots, denoted by $t(t=0,1,2,3 \ldots)$. During a slot $t$, all squares that are numbered $(t \bmod 9)+1$ are allowed to transmit packets simultaneously.

Consider a slot when square $s_{i}$ is allowed to transmit. Then, those squares that may interfere with $s_{i}$ are located along the perimeters of concentric squares centered at $s_{i}$. Since we only allow transmissions between two neighboring squares, at the $j$ th tier, there are at most $8 j$ interfering squares that are at least $(3 j-2) l$ away from the receiver of $s_{i}$. Besides, recall that the network is a rectangle with width $s(n)$ and length $n / s(n)$, where $s(n)=n^{w}$ and $0<w \leq 1 / 2$. Denote the maximum value of $j$ by $J$. Obviously, we have $j \leq J<+\infty$. Thus, with the power propagation model in (1), the cumulative interference at square $s_{i}$, denoted by $I_{i}$, can be calculated as

$$
\begin{aligned}
I_{i} & \leq \sum_{j=1}^{J} 8 j \times \frac{C P(n)}{[1+(3 j-2) l]^{\gamma}} \\
& \leq \sum_{j=1}^{J} 8 j \times \frac{C P(n)}{[(3 j-2) l]^{\gamma}} \\
& \leq \frac{8 C P(n)}{l^{\gamma}}\left[1+\sum_{j=2}^{J}(3 j-2)^{(1-\gamma)}\right] \\
& <\frac{8 C P(n)}{l^{\gamma}}\left[1+\int_{j=0}^{+\infty}(3 j+1)^{(1-\gamma)} d j\right] \\
& =\frac{8 C P(n)}{l^{\gamma}} \cdot \frac{3 \gamma-5}{3 \gamma-6} .
\end{aligned}
$$

We also need a lower bound on the reception power level at the receiver of $s_{i}$, denoted by $R_{i}$. Since the maximum distance for a transmitter to a receiver is $\sqrt{5} l$, we can have

$$
R_{i} \geq \frac{C P(n)}{(1+\sqrt{5} l)^{\gamma}}>\frac{C P(n)}{[(1+\sqrt{5}) l]^{\gamma}} .
$$




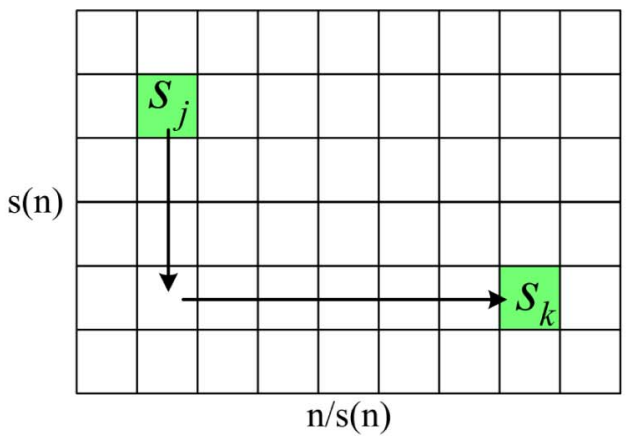

(a)

Fig. 5. The routing protocol used for ad hoc mode transmissions.

As a result, the SINR at the receiver of $s_{i}$, denoted by $S I N R_{i}$, is

$$
\begin{aligned}
S I N R_{i} & =\frac{R_{i}}{N_{0}+I_{i}} \\
& \geq \frac{\frac{C P(n)}{[(1+\sqrt{5}) l]^{\gamma}}}{N_{0}+\frac{8 C P(n)}{l^{\gamma}} \cdot \frac{3 \gamma-5}{3 \gamma-6}},
\end{aligned}
$$

where $N_{0}$ is the ambient noise power at the receiver. By choosing the transmission power $P(n)=c_{3} l^{\gamma}$, where $c_{3}$ $\left(0<c_{3}<+\infty\right)$ is a constant, we can obtain a lower bound on $S I N R_{i}$, i.e.,

$$
S I N R_{i} \geq \frac{c_{3} C}{(1+\sqrt{5})^{\gamma}\left(N_{0}+8 c_{3} C \frac{3 \gamma-5}{3 \gamma-6}\right)},
$$

which is a constant irrespective to the number of nodes $n$. Thus, referring to (2), we find that in every nine time slots, each square has a chance to transmit at a constant transmission rate. As a result, each square in the network can transmit at a constant transmission rate $c_{2} W_{1}$, where $0<c_{2}<+\infty$.

Recall that transmissions in user mode are carried out with only the help of normal nodes. We employ the following routing strategy to relay the packets. Specifically, as shown in Fig. 5a, assume a source node is located in square $s_{j}$ and its destination node is located in square $s_{k}$. Packets from this source node are first relayed along those squares that have the same $x$-coordinate as square $s_{j}$ until they arrive at a square that has the same $y$-coordinate as square $s_{k}$. Then, these packets are relayed along the squares that have the same $y$-coordinate as square $s_{k}$ until they arrive at the destination node.

Consider an arbitrary square $s_{i}$ in the network as shown in Fig. $5 \mathrm{~b}$. Let $N_{x}$ and $N_{y}$ denote the number of source nodes which are located in squares with the same $x$-coordinate as $s_{i}$, and the number of destination nodes which are located in squares with the same $y$-coordinate as $s_{i}$, respectively. Thus, we have

$$
\begin{aligned}
& \mathbb{E}\left[N_{x}\right]=n \cdot \frac{l}{n / s(n)}=n^{w}\left(c_{1} \log n\right)^{\frac{1}{2}}, \\
& \mathbb{E}\left[N_{y}\right]=n^{d} \cdot \frac{l}{s(n)}=n^{d-w}\left(c_{1} \log n\right)^{\frac{1}{2}},
\end{aligned}
$$

and obtain the following lemma:

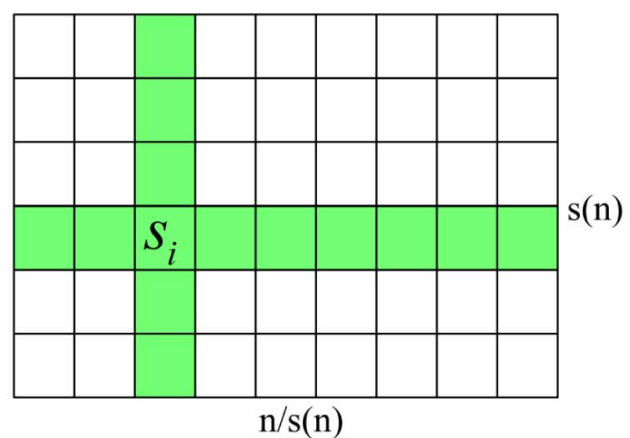

(b)

Lemma 3. For every square, w.h.p.

1. There are at most $2 n^{w}\left(c_{1} \log n\right)^{\frac{1}{2}}$ source nodes which are located in squares with the same $x$-coordinate.

2. The number of destination nodes which are located in squares with the same $y$-coordinate is at most $2 n^{d-w}\left(c_{1} \log n\right)^{\frac{1}{2}}$ when $0<w<d<1$, and at most $c_{4}$ when $0<d<w \leq \frac{1}{2}$, where $c_{4}$ is a constant and $c_{4}>\frac{2}{w-d}$.

Proof. Recall the Chernoff bounds [7]:

- For any $\delta>0$,

$$
\mathbb{P}\left(X_{i}>(1+\delta) \mathbb{E}\left[X_{i}\right]\right)<e^{-\mathbb{E}\left[X_{i}\right] f(\delta)},
$$

where $f(\delta)=(1+\delta) \log (1+\delta)-\delta$.

- For any $0<\delta<1$,

$$
\mathbb{P}\left(X_{i}<(1-\delta) \mathbb{E}\left[X_{i}\right]\right)<e^{-\frac{1}{2} \delta^{2} \mathbb{E}\left[X_{i}\right]} .
$$

1. According to the Chernoff bound in (8), we obtain that

$$
\mathbb{P}\left(N_{x}>2 n^{w}\left(c_{1} \log n\right)^{\frac{1}{2}}\right)<e^{-f(1) n^{w}\left(c_{1} \log n\right)^{\frac{1}{2}}},
$$

where $f(1)=2 \log 2-1>0$. Since $0<w \leq \frac{1}{2}$, as $n \rightarrow \infty$, we have $\mathbb{P}\left(N_{x}>2 n^{w}\left(c_{1} \log n\right)^{\frac{1}{2}}\right) \rightarrow 0$. Let $\mathbb{P}\left(N_{x} \leq 2 n^{w}\left(c_{1} \log n\right)^{\frac{1}{2}} \forall i\right)$ denote the probability that for each square the number of source nodes located in squares with the same $x$-coordinate is at most $2 n^{w}\left(c_{1} \log n\right)^{\frac{1}{2}}$. We can obtain that

$$
\begin{aligned}
& \left.\mathbb{P}\left(N_{x} \leq 2 n^{w}\left(c_{1} \log n\right)^{\frac{1}{2}}\right) \forall i\right) \\
& \geq 1-\frac{n}{c_{1} \log n} \mathbb{P}\left(N_{x}>2 n^{w}\left(c_{1} \log n\right)^{\frac{1}{2}}\right) \\
& >1-\frac{n}{c_{1} \log n} e^{-f(1) n^{w}\left(c_{1} \log n\right)^{\frac{1}{2}}},
\end{aligned}
$$

which approaches 1 as $n \rightarrow \infty$.

2. First, when $0<w<d<1$, we have

$$
\mathbb{P}\left(N_{y}>2 n^{d-w}\left(c_{1} \log n\right)^{\frac{1}{2}}\right)<e^{-f(1) n^{d-w}\left(c_{1} \log n\right)^{\frac{1}{2}}},
$$

which approaches 0 as $n \rightarrow \infty$. Similar to that in 1), we can easily show that $\mathbb{P}\left(N_{y} \leq\right.$ $\left.2 n^{d-w}\left(c_{1} \log n\right)^{\frac{1}{2}} \forall i\right) \rightarrow 1$ as $n \rightarrow \infty$.

Second, when $0<d<w \leq \frac{1}{2}$, according to the Chernoff bound in (8), we can obtain that 


$$
\begin{aligned}
\mathbb{P}\left(N_{y}>(1+\delta) \mathbb{E}\left[N_{y}\right]\right) & <e^{-\mathbb{E}\left[N_{y}\right][(1+\delta) \log (1+\delta)-\delta]} \\
& =\frac{e^{\delta \mathbb{E}\left[N_{y}\right]}}{(1+\delta)^{(1+\delta) \mathbb{E}\left[N_{y}\right]}} .
\end{aligned}
$$

Choose $1+\delta=\frac{c_{4}}{\mathbb{E}\left[N_{y}\right]}=c_{4} n^{w-d}\left(c_{1} \log n\right)^{-\frac{1}{2}}$, where $c_{4}$ is a constant that will be determined later. Then, we have

$$
\begin{aligned}
\mathbb{P}\left(N_{y}>c_{4}\right) & <\frac{e^{\left[c_{4} n^{w-d}\left(c_{1} \log n\right)^{-\frac{1}{2}}-1\right] n^{d-w}\left(c_{1} \log n\right)^{\frac{1}{2}}}}{\left[c_{4} n^{w-d}\left(c_{1} \log n\right)^{-\frac{1}{2}}\right]^{c_{4}}} \\
& =\frac{e^{c_{4}-n^{d-w}\left(c_{1} \log n\right)^{\frac{1}{2}}}}{c_{4}^{c_{4}}\left[n^{w-d}\left(c_{1} \log n\right)^{-\frac{1}{2}}\right]^{c_{4}}} \\
& <\frac{e^{c_{4}}}{c_{4}^{c_{4}}} \cdot\left[n^{d-w}\left(c_{1} \log n\right)^{\frac{1}{2}}\right]^{c_{4}}
\end{aligned}
$$

which approaches 0 as $n \rightarrow \infty$. Besides, we can also obtain that

$$
\begin{aligned}
\mathbb{P}\left(N_{y} \leq c_{4} \forall i\right) & \geq 1-\frac{n}{c_{1} \log n} \mathbb{P}\left(N_{x}>c_{4}\right) \\
& >1-\frac{e^{c_{4}}}{c_{4}^{c_{4}}} n^{(d-w) c_{4}+1}\left(c_{1} \log n\right)^{\frac{1}{2} c_{4}-1} .
\end{aligned}
$$

When we choose $c_{4}>\frac{2}{w-d}$, we can get $(d-w) c_{4}+$ $1<-1$, and hence $\mathbb{P}\left(N_{y} \leq c_{4} \forall \mathrm{i}\right) \rightarrow 1$ as $n \rightarrow \infty . \square$

Besides, in the network we have $n$ source nodes while only $n^{d}$ destination nodes. We can also have a lemma as follows:

Lemma 4. For each destination node, w.h.p., there are at most $2 n^{1-d}$ source nodes destined to it.

Proof. Consider an arbitrary destination node $i$. Let $N_{i}$ be a random variable denoting the number of source nodes that have $i$ as their destination node, and $\mathbb{E}\left[N_{i}\right]$ the expectation of $N_{i}$. Then, we have $\mathbb{E}\left[N_{i}\right]=n \cdot \frac{1}{n^{d}}=n^{1-d}$.

According to the Chernoff bound in (8), we can obtain that

$$
\mathbb{P}\left(N_{i}>2 n^{1-d}\right)<e^{-f(1) n^{1-d}},
$$

where $f(1)>0$ and $1-d>0$. So, $\mathbb{P}\left(N_{i}>2 n^{1-d}\right) \rightarrow 0$ as $n \rightarrow \infty$. Besides, we also have

$$
\begin{aligned}
\mathbb{P}\left(N_{i} \leq 2 n^{1-d} \forall i\right) & \geq 1-n^{d} \mathbb{P}\left(N_{i}>2 n^{1-d}\right) \\
& >1-n^{d} e^{-f(1) n^{1-d}}
\end{aligned}
$$

which approaches 1 as $n \rightarrow \infty$.

Denote the number of flows that cross square $s_{i}$ as $F_{i}$. Since each source node only generates one flow, and there are at most $2 n^{1-d}$ flows for each destination node as shown in Lemma 4, we can obtain that for all $i$,

$$
\begin{aligned}
F_{i} & \leq N_{x}+2 n^{1-d} N_{y} \\
& \leq\left\{\begin{array}{c}
2 n^{w}\left(c_{1} \log n\right)^{\frac{1}{2}}+4 n^{1-w}\left(c_{1} \log n\right)^{\frac{1}{2}}, \\
\text { when } 0<w<d<1, \\
2 n^{w}\left(c_{1} \log n\right)^{\frac{1}{2}}+2 c_{4} n^{1-d}, \\
\text { when } 0<d<w \leq \frac{1}{2},
\end{array}\right.
\end{aligned}
$$

i.e.,

$$
F_{i}=\mathrm{O}\left(\max \left\{n^{w}(\log n)^{\frac{1}{2}}, n^{1-w}(\log n)^{\frac{1}{2}}, n^{1-d}\right\}\right) .
$$

Recall that according to Lemma 2, each square in the network can achieve a constant transmission rate $c_{2} W_{1}$. Thus, from (10), we can obtain

$$
T_{u}=\Omega\left(\min \left\{\frac{n^{-w}}{\sqrt{\log n}} W_{1}, \frac{n^{w-1}}{\sqrt{\log n}} W_{1}, n^{d-1} W_{1}\right\}\right) .
$$

Since $0<w \leq \frac{1}{2}$, we get $-w \geq-\frac{1}{2}$ and $w-1 \leq-\frac{1}{2}$. So, we obtain that

$$
T_{u}=\Omega\left(\min \left\{\frac{n^{w-1}}{\sqrt{\log n}}, n^{d-1}\right\}\right) .
$$

\subsection{Achievable Throughput in Help Mode}

Transmissions in help mode are carried out in three steps: from a source node to the nearest helping node, from this helping node to the helping node nearest to the destination node, and finally from that helping node to the destination node. We analyze the throughput capacity in these three steps, respectively, in the following, and the minimum of these three is an achievable throughput in help mode.

Step 1: From source nodes to the helping network. Recall that in regular heterogeneous wireless networks the helping nodes are regularly placed. So a network can be divided into squares of equal area $1 / m$, which we call cells. We assume source nodes have low transmission power and they need to transmit packets to the helping node in the same cell via multiple hops. Then, we can have the following lemma. The proof is similar to that of Lemma 4.

Lemma 5. In each cell, there are at most $2 n / m$ normal nodes w.h.p., where $m=n^{b}(0<b<1)$.

In Lemma 1, we have shown that in each square, there exists at least one normal node that can help relay traffic. Besides, in Lemma 2, by chopping time into slots and scheduling the transmissions in each slot, we have presented that each square can achieve a constant transmission rate $c_{2} W_{1}$ in user mode. Using the same approach, in help mode we further divide each time slot into two equal minislots: the first for uplink transmissions and the second for downlink transmissions. Then, we can also show that in Step 1 each square can transmit at a constant transmission rate $c_{6} W_{2}$, where $0<c_{6}<+\infty$ is a deterministic constant. Furthermore, by Lemma 5 , in each cell, there are at most $\frac{2 n}{m}$ normal nodes that one square has to carry traffic for. Denote the throughput capacity in Step 1 by $T_{h 1}$. We can obtain that

$$
T_{h 1}=\Omega\left(\frac{m}{n} W_{2}\right)=\Omega\left(n^{b-1}\right) .
$$

Step 2: Helping network relay. Notice that each cell is a big square with length $l^{\prime}=\sqrt{n / m}$, and there is exactly one helping node in each cell. Thus, similar to Lemma 2, we can also have the following lemma:

Lemma 6. Each cell in the network can transmit at a transmission rate $c_{7} W_{4}$, where $c_{7}$ is a deterministic positive constant.

Besides, as shown in Fig. 6, notice that the $m$ big squares with length $l^{\prime}=\sqrt{n / m}$ cannot cover the whole network 


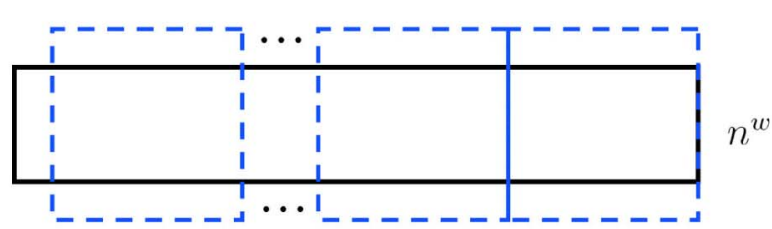

Since $n^{1-w} / n^{w}=\Omega(m)$,

$$
\text { (a) } l=\sqrt{n / m} \text {. }
$$

Fig. 6. A special case for covering the network with $m$ squares.

when $s(n)=o\left(l^{\prime}\right)$, i.e., $w+b / 2<1 / 2$. In this case, we choose $l^{\prime}=n^{1-w-b}$ to ensure full network coverage. Note that when $w+b / 2=1 / 2$, we have $\sqrt{1 / m}=n^{1-w-b}$. As a result, we have the following lemma.

Lemma 7. For every cell, w.h.p.

1. The number of source nodes which are located in the cells with the same $x$-coordinate, denoted by $C_{x}$, is at most $2 n^{w-\frac{b}{2}+\frac{1}{2}}$ when $w+\frac{b}{2}-\frac{1}{2}>0$, and at most $2 n^{1-b}$ when $w+\frac{b}{2}-\frac{1}{2} \leq 0$.

2. The number of destination nodes which are located in the cells with the same $y$-coordinate, denoted by $C_{y}$, is at most $2 n^{d-w-\frac{b}{2}+\frac{1}{2}}$ when $d>w+\frac{b}{2}-\frac{1}{2}>0$, at most $c_{8}$ when $w+\frac{b}{2}-\frac{1}{2}>d>0$, where $c_{8}$ is a constant and $c_{8}>\frac{4}{2 w+b-2 d-1}$, and at most $n^{d}$ when $w+\frac{b}{2}-\frac{1}{2} \leq 0$.

Proof. When $w+\frac{b}{2}>\frac{1}{2}$, the proof is similar to that in Lemma 3. When $w+\frac{b}{2} \leq \frac{1}{2}$, there is only one cell for each $x$-coordinate, and hence the number of source nodes is the same as shown in Lemma 5. Besides, all the cells have the same $y$-coordinate. So the number of destination nodes located in the cells with the same $y$-coordinate is at most the total number of destination nodes in the network, i.e., $n^{d}$.

Denote the number of flows that cross an arbitrary cell $C_{i}$ as $B_{i}$, where $i \in[1, m]$. Remember that each source node only generates one flow, and that there are at most $2 n^{1-d}$ flows for each destination node as shown in Lemma 4. Thus, we can obtain that for any $i$,

$$
\begin{aligned}
B_{i} & \leq C_{x}+2 n^{1-d} C_{y} \\
& \leq\left\{\begin{array}{l}
2 n^{w-\frac{b}{2}+\frac{1}{2}}+4 n^{-w-\frac{b}{2}+\frac{3}{2}}, \quad \text { when } 0<w+\frac{b}{2}-\frac{1}{2}<d<1, \\
2 n^{w-\frac{b}{2}+\frac{1}{2}}+2 c_{3} n^{1-d}, \quad \text { when } 0<d<w+\frac{b}{2}-\frac{1}{2}<1, \\
2 n^{1-b}+n, \quad \text { when } w+\frac{b}{2}-\frac{1}{2} \leq 0 .
\end{array}\right.
\end{aligned}
$$

Denote the throughput capacity in Step 2 by $T_{h 2}$. Thus, from Lemma 6, we can obtain that

$$
\begin{aligned}
T_{h 2} & =\Omega\left(\min \left\{n^{-w+\frac{b}{2}-\frac{1}{2}} W_{4}, n^{I\left(w+\frac{b}{2}-\frac{1}{2}\right)-1} W_{4}, n^{d-1} W_{4}\right\}\right) \\
& =\Omega\left(\min \left\{n^{I\left(w+\frac{b}{2}-\frac{1}{2}\right)-1} W_{4}, n^{d-1} W_{4}\right\}\right),
\end{aligned}
$$

where $I(\cdot)$ is a function, and $I(x)=x$ if $x>0$, and $I(x)=0$ if $x \leq 0$.

Step 3: From the helping network to destination nodes. We first give a lemma that will be used later.

Lemma 8. In each cell, w.h.p., there are at most $2 n^{d-b}$ destination nodes when $0<b<d<1$, and at most $c_{9}$, where $c_{9}>\frac{1+b}{b-d}$, destination nodes when $0<d<b<1$.

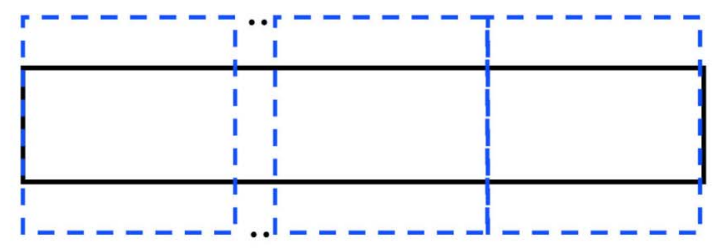

we choose $l^{\prime}=n^{1-w} / m=n^{1-w-b}$.

(b) $l^{\prime}=n^{1-w-b}$.

Proof. Consider cell $i$. Let $Y_{i}$ be a random variable denoted as the number of destination nodes in cell $i$, and $\mathbb{E}\left[Y_{i}\right]$ the expectation of $Y_{i}$. Then, we have $\mathbb{E}\left[Y_{i}\right]=\frac{n^{d}}{m}=n^{d-b}$.

1. $0<b<d<1$. According to the Chernoff bound, we can obtain that

$$
\mathbb{P}\left(Y_{i}>2 n^{d-b}\right)<e^{-c_{10} n^{d-b}},
$$

where $c_{10}=f(1)=2 \log 2-1>0$. Thus, as $n \rightarrow \infty$, we have $\mathbb{P}\left(Y_{i}>2 n^{d-b}\right) \rightarrow 0$. Besides, the probability that the number of destination nodes is at most $2 n^{d-b}$ in all cells, denoted by $\mathbb{P}\left(Y_{i} \leq 2 n^{d-b} \forall i\right)$, can be calculated as

$$
\begin{aligned}
\mathbb{P}\left(Y_{i} \leq 2 n^{d-b} \forall i\right) & \geq 1-m \mathbb{P}\left(Y_{i}>2 n^{d-b}\right) \\
& >1-n^{b} e^{-c_{10} n^{d-b}}
\end{aligned}
$$

which approaches 1 as $n \rightarrow \infty$.

2. $0<d<b<1$. Again, according to the Chernoff bound introduced before, we can obtain that

$$
\begin{aligned}
\mathbb{P}\left(Y_{i}>(1+\delta) \mathbb{E}\left[Y_{i}\right]\right) & <e^{-\mathbb{E}\left[Y_{i}\right][(1+\delta) \log (1+\delta)-\delta]} \\
& =\frac{e^{\delta \mathbb{E}\left[Y_{i}\right]}}{(1+\delta)^{(1+\delta) \mathbb{E}\left[Y_{i}\right]}}
\end{aligned}
$$

Let $1+\delta=\frac{c_{9}}{\mathbb{E}\left[Y_{i}\right]}=c_{9} n^{b-d}$, where $c_{3}$ is a constant that will be determined later. Then, we have

$$
\begin{aligned}
\mathbb{P}\left(Y_{i}>c_{9}\right) & <\frac{e^{\left(c_{9} n^{b-d}-1\right) n^{d-b}}}{\left(c_{9} n^{b-d}\right)^{c_{9}}} \\
& =\frac{e^{c_{9}-n^{d-b}}}{c_{9}^{c_{9}} n^{c_{9}(b-d)}} \\
& <\frac{e^{c_{9}}}{c_{9}^{c_{9}}} \cdot n^{c_{9}(d-b)} \rightarrow 0 \text { as } \mathrm{n} \rightarrow \infty .
\end{aligned}
$$

Besides, we can also obtain that

$$
\begin{aligned}
\mathbb{P}\left(Y_{i} \leq c_{9} \forall i\right) & \geq 1-m \mathbb{P}\left(Y_{i}>c_{9}\right) \\
& >1-n^{b} \frac{e^{c_{9}}}{c_{9}^{c_{9}}} \cdot n^{c_{9}(d-b)} \\
& =1-\frac{e^{c_{9}}}{c_{9}^{c_{9}}} \cdot n^{c_{9} d-\left(c_{9}-1\right) b} .
\end{aligned}
$$

When we choose $c_{9}>\frac{1+b}{b-d}$, we can get $c_{9} d-\left(c_{9}-\right.$ $1) b<-1$, and hence $\mathbb{P}\left(Y_{i} \leq c_{9} \forall i\right) \rightarrow 1$ as $n \rightarrow \infty$. $\square$ 
We let the helping nodes use the same transmission power as the normal nodes to deliver packets to destination nodes within the cells in multihop fashion. As we mentioned in Step 1, the squares scheduled for uplink transmissions in the first mini-slot of a time slot can carry out downlink transmissions in the second mini-slot. Thus, each square engaged in the downlink transmissions in Step III can also have a constant transmission rate $c_{11} W_{3}$, where $c_{11}\left(0<c_{11}<+\infty\right)$ is a deterministic constant. ${ }^{1}$ In addition, from Lemmas 4 and 8, we find that when $0<b<d<1$, in each cell, the number of flows from a helping node to destination nodes is at most $2 n^{d-b} \times 2 n^{1-d}$ w.h.p., i.e., $4 n^{1-b}$, and when $0<d<b<1$, in each cell, the number of flows from a helping node to destination nodes is at most $c_{9} \times$ $2 n^{1-d}$ w.h.p., i.e., $2 c_{9} n^{1-d}$. Denote the throughput capacity in Step 3 by $T_{h 3}$. Then, we can obtain that

$$
T_{h 3}= \begin{cases}\Omega\left(n^{b-1} W_{3}\right), & \text { when } 0<b<d<1 \\ \Omega\left(n^{d-1} W_{3}\right), & \text { when } 0<d<b<1 .\end{cases}
$$

Thus, combining (12), (13), and (14), we can get

$$
\begin{aligned}
T_{h} & =\Omega\left(\min \left\{T_{h 1}, T_{h 2}, T_{h 3}\right\}\right) \\
& =\Omega\left(\min \left\{n^{b-1}, n^{I\left(w+\frac{b}{2}-\frac{1}{2}\right)-1} W_{4}, n^{d-1}\right\}\right) .
\end{aligned}
$$

\subsection{An Achievable Throughput in Regular Heterogeneous Wireless Networks}

Substituting the results in (11) and (15) into (3), we can have the following theorem:

Theorem 1. An achievable throughput in regular heterogeneous wireless networks, denoted by $T$, is

$$
\begin{aligned}
T=\Omega\left(\operatorname { m a x } \left\{\min \left\{\frac{n^{w-1}}{\sqrt{\log n}}, n^{d-1}\right\},\right.\right. \\
\left.\left.\min \left\{n^{b-1}, n^{I\left(w+\frac{b}{2}-\frac{1}{2}\right)-1} W_{4}, n^{d-1}\right\}\right\}\right),
\end{aligned}
$$

when $0<w \leq \frac{1}{2}, 0<b<1$, and $0<d<1$.

\section{Throughput CAPACITY OF RANDOM Heterogeneous Wireless Networks}

In this section, we study the throughput capacity of random heterogeneous wireless networks with uniformly and independently placed helping nodes when $0<w \leq \frac{1}{2}$, $0<b<1$, and $0<d<1$. Similarly, we also need to find an achievable throughput in user mode and in help mode, denoted by $T_{u}^{\prime}$ and $T_{h}^{\prime}$, respectively. Then, an achievable throughput in random heterogeneous wireless networks, denoted by $T^{\prime}$, can be obtained by choosing the maximum of these two, i.e.,

$$
T^{\prime}=\max \left\{T_{u}^{\prime}, T_{h}^{\prime}\right\} .
$$

Notice that $T_{u}^{\prime}$ is the same as $T_{u}$ shown in (11), but $T_{h}^{\prime}$ will be different from $T_{h}$ due to random distribution of the helping nodes. In the following, we present how to find $T_{h}^{\prime}$.

Recall the definition of Voronoi Tessellation: given a set of $m$ points in a plane, Voronoi tessellation divides the domain

1. Helping nodes' network interfaces working on normal nodes' frequency band will be considered as normal nodes when scheduling their transmissions. into a set of polygonal regions, the boundaries of which are the perpendicular bisectors of the lines joining the points. It has been shown in [14, Lemma 4.1] that for every $\varepsilon>0$, there is a Voronoi tessellation with the property that every Voronoi cell contains a disk of radius $\varepsilon$ and is contained in a disk of radius $2 \varepsilon$. Then, for the $m$ base stations in a dense network with area 1, we can construct a Voronoi tessellation $V_{n}$ for which

- (V1) Every Voronoi cell contains a disk of area $\frac{100 n \log m}{m}$.

- (V2) Every Voronoi cell is contained in a disk of radius $2 \rho(n)$, where $\rho(n):=$ the radius of a disk of area $\frac{100 n \log m}{m}$.

In this case, we consider each Voronoi cell as a cell in the network. In each cell, we randomly choose one helping node as the helping node of the cell. The other helping nodes help relay the packets in the helping network.

Similar to that in Section 3.2, the transmissions in help mode are also carried out in three steps as follows:

Step 1: From source nodes to the helping network. We also assume source nodes have low transmission power and they need to transmit packets to helping nodes via multiple hops. Thus, we can have the following result. The proof is similar to that of Lemma 4.

Lemma 9. In each Voronoi cell, there are at most $\frac{1,200 \mathrm{n} \log m}{m}$ normal nodes w.h.p.

Denote the throughput capacity in Step 1 by $T_{h 1}^{\prime}$. Since each node is a source node, along the line in Section 3.2, we can obtain that

$$
T_{h 1}^{\prime}=\Omega\left(\frac{m}{n \log m} W_{2}\right)=\Omega\left(\frac{n^{b-1}}{\log n}\right) .
$$

Step 2: Helping network relay. When $w+\frac{b}{2}>\frac{1}{2}$, we further divide the network into big squares with length $l^{\prime \prime}=\sqrt{c_{12} n \log m / m}$, where $c_{12}>1 / b$ is a constant. When $w+\frac{b}{2} \leq \frac{1}{2}$, we make $l^{\prime \prime}=c_{12} n^{1-w-b} \log m$, where $c_{12}>1 / b$. Then, we can have the following lemma.

Lemma 10. In random heterogeneous wireless networks

1. Every big square has at least one helping node in it w.h.p.

2. Each big square in the network can transmit at a constant transmission rate $c_{13} W_{2}$, where $0<c_{13}<$ $+\infty$ is a deterministic constant.

Besides, similar to Lemma 7, we can have the following lemma:

Lemma 11. For every big square, w.h.p.,

1. The number of source nodes from which the traffic goes through the big square, denoted by $C_{x}^{\prime}$ is $\mathrm{O}\left(n^{w-\frac{b}{2}+\frac{1}{2}}(\log n)^{\frac{1}{2}}\right)$ whe $n \quad w+\frac{b}{2}-\frac{1}{2}>0$, a nd $\mathrm{O}\left(n^{1-b} \log n\right)$ when $w+\frac{b}{2}-\frac{1}{2} \leq 0$.

2. The number of destination nodes to which the traffic goes through the big square, denoted by $C_{y^{\prime}}^{\prime}$ is $\mathrm{O}\left(n^{d-w-\frac{b}{2}+\frac{1}{2}}(\log n)^{\frac{1}{2}}\right)$ when $d>w+\frac{b}{2}-\frac{1}{2}>0$, at most $c_{14}$ when $w+\frac{b}{2}-\frac{1}{2}>d>0$, where $c_{14}$ is a 

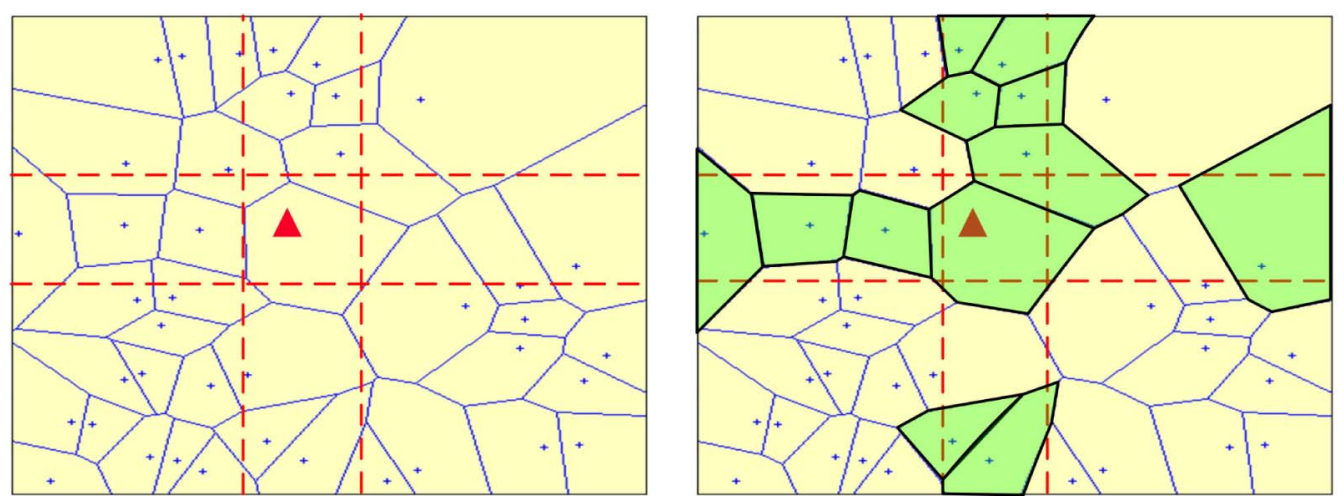

Fig. 7. A Voronoi tessellation example. +'s stands for the helping nodes of each cell, and we have replaced two points with the helping nodes in the figure.

constant and $c_{14}>\frac{4}{2 w+b-2 d-1}$, and at most $n^{d}$ when $w+\frac{b}{2}-\frac{1}{2} \leq 0$.

Proof. Notice that in random heterogeneous wireless networks, the expectation of $C_{x}^{\prime}$ and $C_{y}^{\prime}$ cannot be directly calculated as in (6) and (7), respectively. For example, as shown in Fig. $7, C_{x}^{\prime}$ is not simply the number of source nodes located in big squares with the same $x$-coordinate as the current one because $C_{x}^{\prime}$ may include some source nodes outside such big squares and not include some source nodes inside such big squares. Thus, for a big square, the expectation of $C_{x}^{\prime}$ should be the average number of helping nodes (of the cells) in big squares with the same $x$-coordinate times the average number of source nodes associated with each big square. The expectation of $C_{y}^{\prime}$ should be derived similarly. Therefore, we have

$$
\begin{aligned}
& \mathbb{E}\left[C_{x}^{\prime}\right]=\Theta\left(\frac{m}{\log m}\right) \cdot \frac{l^{\prime \prime}}{n / s(n)} \cdot \Theta\left(\frac{n \log m}{m}\right)=\Theta\left(l^{\prime \prime} s(n)\right), \\
& \mathbb{E}\left[C_{y}^{\prime}\right]=\Theta\left(\frac{m}{\log m}\right) \cdot \frac{l^{\prime \prime}}{s(n)} \cdot \Theta\left(\frac{n^{d} \log m}{m}\right)=\Theta\left(\frac{n^{d} l^{\prime \prime}}{s(n)}\right) .
\end{aligned}
$$

The rest of the proof follows that in Lemma 7.

Denote the number of flows that cross an arbitrary big square $S_{i}$ as $B_{i}^{\prime}$. We can obtain that for any $i$,

$$
\begin{aligned}
B_{i}^{\prime} \leq & C_{x}^{\prime}+2 n^{1-d} C_{y}^{\prime} \\
= & \left\{\begin{array}{c}
\mathrm{O}\left(n^{w-\frac{b}{2}+\frac{1}{2}}(\log n)^{\frac{1}{2}}\right)+\mathrm{O}\left(n^{-w-\frac{b}{2}+\frac{3}{2}}(\log n)^{\frac{1}{2}}\right) \\
\text { when } d>w+\frac{b}{2}-\frac{1}{2}>0, \\
\mathrm{O}\left(n^{w-\frac{b}{2}+\frac{1}{2}}(\log n)^{\frac{1}{2}}\right)+\mathrm{O}\left(n^{1-d}\right), \\
\text { when } w+\frac{b}{2}-\frac{1}{2}>d>0, \\
\mathrm{O}\left(n^{1-b} \log n\right)+\mathrm{O}(n), \quad \text { when } w+\frac{b}{2}-\frac{1}{2}<0 .
\end{array}\right.
\end{aligned}
$$

Denote the throughput capacity in Step 2 by $T_{h 2}^{\prime}$. Thus, we can obtain that

$$
\begin{aligned}
T_{h 2}^{\prime} & =\min \left\{\frac{n^{-w+\frac{b}{2}-\frac{1}{2}}}{(\log n)^{\frac{1}{2}}} W_{4}, \frac{n^{I\left(w+\frac{b}{2}-\frac{1}{2}\right)-1}}{(\log n)^{J\left(w+\frac{b}{2}-\frac{1}{2}\right)}} W_{4}, n^{d-1} W_{4}\right\} \\
& =\min \left\{\frac{n^{I\left(w+\frac{b}{2}-\frac{1}{2}\right)-1}}{(\log n)^{J\left(w+\frac{b}{2}-\frac{1}{2}\right)}} W_{4}, n^{d-1} W_{4}\right\},
\end{aligned}
$$

where $J(\cdot)$ is a function, and $J(x)=\frac{1}{2}$ when $x>0$, and $J(x)=0$ when $x \leq 0$.
Step 3: From the helping network to destination nodes. The same as that in regular heterogeneous wireless networks, we set helping nodes' transmission power to be the same as normal nodes'. Then, we can also show that in Step 3 each square can have a constant transmission rate $c_{15} W_{3}$, where $c_{15}\left(0<c_{15}<+\infty\right)$ is a deterministic constant. Besides, similar to Lemma 8, we can have the following lemma:

Lemma 12. In each cell, w.h.p., the number of destination nodes is $\mathrm{O}\left(n^{d-b} \log n\right)$ when $0<b<d<1$, and $\mathrm{O}\left(c_{9}\right)$, where $c_{9}>$ $\frac{1+b}{b-d}$ when $0<d<b<1$.

Denote the throughput capacity in Step 3 by $T_{h 3}^{\prime}$. Recall Lemma 4, and we can obtain that

$$
T_{h 3}^{\prime}=\left\{\begin{array}{l}
\Omega\left(n^{b-1} W_{3} / \log n\right), \quad \text { when } 0<b<d<1 \\
\Omega\left(n^{d-1} W_{3}\right), \quad \text { when } 0<d<b<1
\end{array}\right.
$$

Thus, combining (17), (18), and (19), we can obtain

$$
\begin{aligned}
T_{h}^{\prime} & =\Omega\left(\min \left\{T_{h 1}^{\prime}, T_{h 2}^{\prime}, T_{h 3}^{\prime}\right\}\right) \\
& =\Omega\left(\min \left\{\frac{n^{b-1}}{\log n}, \frac{n^{I\left(w+\frac{b}{2}-\frac{1}{2}\right)-1}}{(\log n)^{J\left(w+\frac{b}{2}-\frac{1}{2}\right)}} W_{4}, n^{d-1}\right\}\right) .
\end{aligned}
$$

Substituting the results in (20) into (16), we can have the following theorem:

Theorem 2. An achievable throughput in random heterogeneous wireless networks, denoted by $T^{\prime}$, is

$$
\begin{aligned}
T^{\prime}=\Omega\left(\operatorname { m a x } \left\{\min \left\{\frac{n^{w-1}}{\sqrt{\log n}}, n^{d-1}\right\},\right.\right. \\
\left.\left.\min \left\{\frac{n^{b-1}}{\log n}, \frac{n^{I\left(w+\frac{b}{2}-\frac{1}{2}\right)-1}}{(\log n)^{J\left(w+\frac{b}{2}-\frac{1}{2}\right)}} W_{4}, n^{d-1}\right\}\right\}\right) .
\end{aligned}
$$

when $0<w \leq \frac{1}{2}, 0<b<1$, and $0<d<1$.

\section{More Results in Extreme Cases}

Sections 3 and 4 present the capacity of regular and random heterogeneous wireless networks, respectively, when $0<w \leq \frac{1}{2}, 0<b<1$, and $0<d<1$. In this section, we discuss some extreme cases, i.e., when $w=0, b=0$ or 1 , and $d=0$ or 1 . 


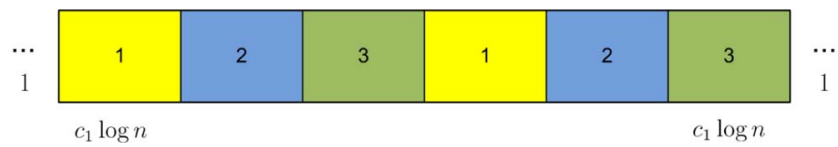

Fig. 8. Squares in the network when $w=0$.

\subsection{Extreme Cases in Regular Heterogeneous Wireless Networks}

We first look into the extreme cases in regular networks. In user mode, when $w=0$, we divide the network into rectangles with width $s(n)=1$ and length $l=c_{1} \log n$ as shown in Fig. 8 (recall that $c_{1}>1$ is a constant). Then, similar to Lemma 1 , we can show that every square contains at least one normal node w.h.p.

Besides, we divide the network into groups each of which has three rectangles as in Fig. 8. The rectangles in each group are numbered in the same way, and at time slot $t(t=0,1,2,3, \ldots)$ the rectangles numbered $(t \bmod 3)+1$ transmit simultaneously. Following Lemma 2, we can get that the cumulative interference for an arbitrary transmission satisfies

$$
\begin{aligned}
I_{i} & \leq \sum_{j=1}^{n / \log n} 2 j \times \frac{C P(n)}{[1+(3 j-2) l]^{\gamma}} \\
& <\frac{2 C P(n)}{l^{\gamma}}\left[1+\int_{j=0}^{+\infty}(3 j+1)^{(1-\gamma)} d j\right] \\
& =\frac{2 C P(n)}{l^{\gamma}} \cdot \frac{3 \gamma-5}{3 \gamma-6},
\end{aligned}
$$

and the reception power at the receiver is approximately

$$
R_{i} \geq \frac{C P(n)}{(1+2 l)^{\gamma}}>\frac{C P(n)}{(3 l)^{\gamma}},
$$

since $l=\omega(s(n))$. As a result, the SINR at the receiver can be calculated as

$$
\begin{aligned}
S I N R_{i} & \geq \frac{\frac{C P(n)}{(3 l)^{\gamma}}}{N_{0}+\frac{2 C P(n)}{l^{\gamma}} \cdot \frac{3 \gamma-5}{3 \gamma-6}} \\
& =\frac{c_{16} C}{3^{\gamma}\left(N_{0}+2 c_{16} C \frac{3 \gamma-5}{3 \gamma-6}\right)},
\end{aligned}
$$

i.e., lower bounded by a constant, by choosing $P(n)=c_{16} l^{\gamma}$. Thus, each square can transmit at a constant transmission rate $c_{17} W_{1}$, where $0<c_{17}<+\infty$.

Note that when $w=0$, each square has to relay packets for at most all the $n$ flows in the network. Therefore, the user mode per-node throughput is

$$
T_{u}=\Omega\left(\frac{1}{n} W_{1}\right)=\Omega\left(\frac{1}{n}\right),
$$

when $W_{1}=\Theta(1)$.

In help mode, following the processes in Section 3.2, we can find that when $w=0$,

$$
T_{h}=\Omega\left(\min \left\{n^{b-1}, n^{-1} W_{4}, n^{d-1}\right\}\right),
$$

for $0 \leq b<1$. Note that this result is consistent with that in (15) since $w+b / 2-1 / 2=(b-1) / 2 \leq 0$. When $b=1$, it has been shown in [16] that the maximum number of users in each cell is $\log n / \log \log n$. So, for $b=1$ we have that

$$
T_{h}=\Omega\left(\min \left\{n^{b-1} \log \log n / \log n, n^{-1} W_{4}, n^{d-1}\right\}\right) .
$$

Note that these results are obtained when $b \neq d$ according to Lemma $8 .^{2}$ When $b=d$, similarly, we can know that the maximum number of destination nodes in each cell is on the order of $\log n / \log \log n$, and hence the maximum number of downlink flows in each cell is on the order of $n^{1-d} \log n / \log \log n$. Thus, similar to (15), for $b=d$, the help mode throughput is as follows:

$$
T_{h}=\Omega\left(\min \left\{\frac{n^{b-1}}{\log n / \log \log n}, n^{I\left(w+\frac{b}{2}-\frac{1}{2}\right)-1} W_{4}, n^{d-1}\right\}\right) .
$$

Combining the above results with Theorem 1, we have the following result:

Theorem 3. An achievable throughput in regular heterogeneous wireless networks, denoted by $T$, is

$$
\begin{aligned}
T= & \Omega\left(\operatorname { m a x } \left\{\min \left\{\frac{n^{w-1}}{(\sqrt{\log n})^{I^{\prime}(w)}}, n^{d-1}\right\},\right.\right. \\
& \left.\left.\min \left\{\frac{n^{b-1}}{\left(\frac{\log n}{\log \log n}\right)^{I^{\prime \prime}(b, d)}}, n^{I\left(w+\frac{b}{2}-\frac{1}{2}\right)-1} W_{4}, n^{d-1}\right\}\right\}\right) .
\end{aligned}
$$

for any $0 \leq w \leq \frac{1}{2}, 0 \leq b \leq 1$, and $0 \leq d \leq 1$, where

$$
I(x)= \begin{cases}x, & \text { if } x>0 \\ 0, & \text { otherwise }\end{cases}
$$

and

$$
I^{\prime}(x)= \begin{cases}1, & \text { if } x>0 \\ 0, & \text { otherwise }\end{cases}
$$

and

$$
I^{\prime \prime}(x, y)= \begin{cases}0, & \text { if } 0 \leq x<1, \text { and } x \neq y \\ 1, & \text { if } \mathrm{x}=1 \text { or } \mathrm{x}=\mathrm{y}\end{cases}
$$

\subsection{Extreme Cases in Random Heterogeneous Wireless Networks}

We then look into the extreme cases in random networks. The user mode throughput is the same as that in Section 5.1. Here, we focus on the help mode throughput.

When $w=0$ and $b=0$, we can get that

$$
T_{h}^{\prime}=\Omega\left(\min \left\{n^{b-1}, n^{-1} W_{4}, n^{d-1}\right\}\right) .
$$

When $w=0$ and $0<b \leq 1$, it can be easily shown that different from that in regular networks, the number of users in each cell in random networks is at most on the order of $n^{1-b} \log n$. Thus, we can have

2. Both Lemmas 3 and 7 leave a special case out when $w=d$ and when $w+b / 2-1 / 2=d$, respectively. But the results in these two special cases can be derived in the same way as shown here. Since presenting the results in these two special cases does not introduce any new technical merit and will make the final results in Theorem 3 in a very complicated form, we choose to ignore them. Due to the same reason, we will also neglect the special case in Lemma 11 in the discussions for Theorem 4. 


$$
T_{h}^{\prime}=\Omega\left(\min \left\{\frac{n^{b-1}}{\log n}, n^{-1} W_{4}, n^{d-1}\right\}\right),
$$

which is consistent with that in (20).

Besides, these results are obtained when $b \neq d$ according to Lemma 12. When $b=d$, we can know that that the maximum number of destination nodes in each cell is on the order of $\log n$, and hence the maximum number of downlink flows in each cell is on the order of $n^{1-d} \log n$. Thus, for $b=d$, the help mode throughput is the same as that shown above.

Combining the above results with Theorem 2, we have the following result:

Theorem 4. An achievable throughput in random heterogeneous wireless networks, denoted by $T^{\prime}$, is

$$
\begin{aligned}
T^{\prime}= & \Omega\left(\operatorname { m a x } \left\{\min \left\{\frac{n^{w-1}}{(\sqrt{\log n})^{I^{\prime}(w)}}, n^{d-1}\right\},\right.\right. \\
& \left.\left.\min \left\{\frac{n^{b-1}}{(\log n)^{J^{\prime}(b)}}, \frac{n^{I\left(w+\frac{b}{2}-\frac{1}{2}\right)-1}}{(\log n)^{J\left(w+\frac{b}{2}-\frac{1}{2}\right)}} W_{4}, n^{d-1}\right\}\right\}\right) .
\end{aligned}
$$

for any $0 \leq w \leq \frac{1}{2}, 0 \leq b \leq 1$, and $0 \leq d \leq 1$, where

$$
I(x)= \begin{cases}x, & \text { if } x>0 \\ 0, & \text { otherwise }\end{cases}
$$

and

$$
J(x)=\left\{\begin{array}{cl}
1 / 2, & \text { if } x>0, \\
0, & \text { otherwise }
\end{array}\right.
$$

and

$$
J^{\prime}(x)= \begin{cases}0, & \text { if } x=0 \\ 1, & \text { if } 0<x \leq 1\end{cases}
$$

\section{INSIGHTS INTO OUR RESULTS}

From Theorems 3 and 4, we can find that the number of destination nodes, the number of helping nodes, the shape of the network area, and the bandwidth of helping nodes all have significant impacts on the achievable throughput in heterogeneous wireless networks. Notice that the user mode achievable throughput is in fact the throughput of traditional static homogeneous ad hoc networks. Thus, one question is: under what conditions can we have higher throughput in heterogeneous wireless networks than in homogeneous ad hoc networks with the help of some more powerful wireless nodes? We address this question in the following by ignoring the logarithmic factors.

Case 1: $w-d \geq 0$. In this case, we have $(w-1)-(d-1) \geq 0$, which means the user mode throughput is limited by the number of destinations, i.e., $T_{u}=\Omega\left(n^{d-1}\right)$. Thus, no matter how many more powerful helping nodes there are and how much bandwidth they have, heterogeneous networks cannot have higher throughput than homogeneous networks in the order sense.

Case 2: $w-d<0$. In this case, we have $(w-1)-(d-$ 1) $<0$ and hence $T_{u}=\Omega\left(n^{w-1}\right)$. Let $W_{4}=n^{x}$.
1. When $w+\frac{b}{2}-\frac{1}{2} \leq 0$, we get

$$
T_{h}=\Omega\left(\min \left\{n^{b-1}, n^{x-1}, n^{d-1}\right\}\right) .
$$

Then, in order to obtain exponentially higher throughput than homogeneous networks, we need

$$
\left\{\begin{array}{l}
b-1>w-1 \\
x-1>w-1 .
\end{array}\right.
$$

2. When $w+\frac{b}{2}-\frac{1}{2}>0$, we can obtain that

$$
T_{h}=\Omega\left(\min \left\{n^{b-1}, n^{w+\frac{b}{2}+x-\frac{3}{2}}, n^{d-1}\right\}\right) .
$$

Thus, in order to achieve exponentially higher throughput than homogeneous networks, we need

$$
\left\{\begin{array}{l}
b-1>w-1 \\
w+b / 2+x-\frac{3}{2}>w-1 .
\end{array}\right.
$$

To summarize, we have the following corollary:

Corollary 1. The conditions under which heterogeneous networks can have higher throughput than homogeneous networks in the order sense are

- $W_{4}=\omega\left(n^{w}\right)$ when $w+b / 2-1 / 2 \leq 0$ and $w-$ $\min \{b, d\}<0$, or

- $W_{4}=\omega\left(n^{\frac{1-b}{2}}\right)$ when $w+b / 2-1 / 2>0$ and $w-$ $\min \{b, d\}<0$.

Moreover, based on Theorems 3 and 4, we can find that it does not mean that we can have higher throughput as long as the powerful nodes have more bandwidth available. The throughput improvement is also limited by the number of powerful nodes and the number of base stations in the network. Specifically, by letting $W_{4}=n^{x}$,

1. When $w+\frac{b}{2}-\frac{1}{2} \leq 0$, we have

$$
x \leq b-1-(-1) \text { and } x \leq d-1-(-1),
$$

i.e.,

$$
x \leq \min \{b, d\} .
$$

2. When $w+\frac{b}{2}-\frac{1}{2}>0$, we get

$$
\begin{gathered}
\qquad\left\{\begin{array}{l}
x \leq b-1-\left(w+\frac{b}{2}-\frac{3}{2}\right), \\
x \leq d-1-\left(w+\frac{b}{2}-\frac{3}{2}\right),
\end{array}\right. \\
\text { i.e., } \quad x \leq \min \left\{\frac{b}{2}-w+\frac{1}{2}, d-\frac{b}{2}-w+\frac{1}{2}\right\} .
\end{gathered}
$$

In summary, we can have the following result:

Corollary 2. In heterogeneous wireless networks, network throughput may be improved as the bandwidth of helping nodes increases only when

- $W_{4}=\mathrm{O}\left(\min \left\{n^{b}, n^{d}\right\}\right)$ when $w+b / 2-1 / 2 \leq 0$, or

- $W_{4}=\mathrm{O}\left(\min \left\{n^{\frac{b}{2}-w+\frac{1}{2}}, n^{d-\frac{b}{2}-w+\frac{1}{2}}\right\}\right)$ when $w+b / 2-$ $1 / 2>0$. 


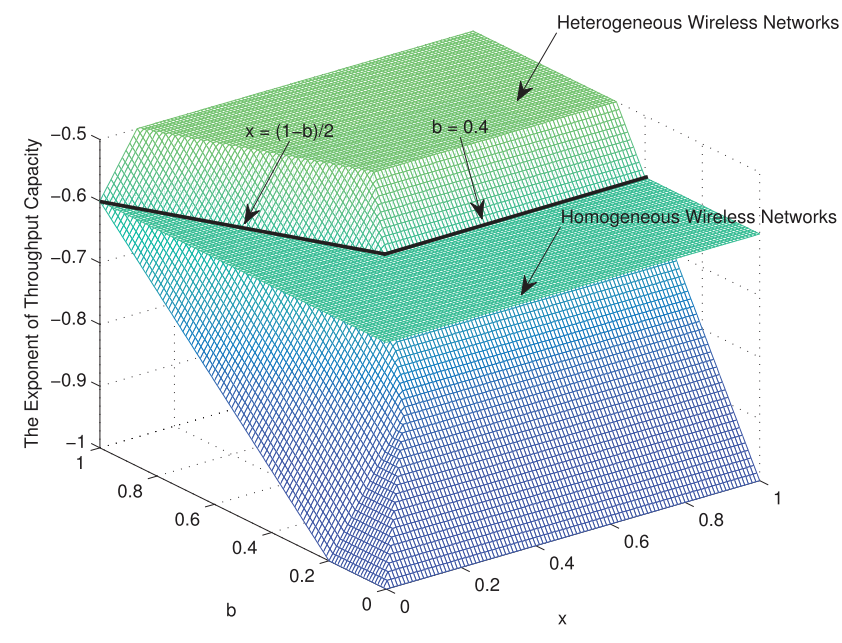

(a) $w=0.4$ and $d=0.5$

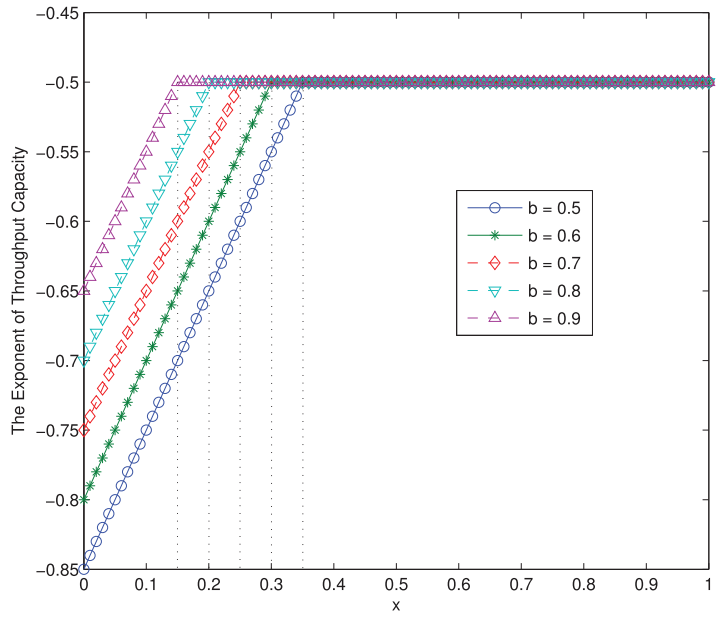

(b) $w=0.4, d=0.5$, and $b=0.5 \sim 0.9$

Fig. 9. Simulation results when $w=0.4$ and $d=0.5$. (a) The throughput capacity of homogeneous wireless networks and of heterogeneous wireless networks. (b) The throughput capacity of heterogeneous wireless networks when $b=0.5,0.6,0.7,0.8$, and 0.9 , respectively.

To better illustrate the results obtained above, we present in the following some numerical results based on the theoretical throughput capacity results shown in Theorems 3 and 4 . Consider a network with $w=0.4$ and $d=0.5$. According to Theorems 3 and 4 , we compare the throughput capacity of heterogeneous wireless networks with that of homogeneous wireless networks in Fig. 9a. We use the exponents of the throughput capacity instead of the throughput capacity itself to show the results more clearly. On the other hand, according to Corollary 1, in order to achieve higher throughput in heterogeneous networks than in homogeneous networks, we need 1) $x>0.4$ when $b \leq 0.2$ and $0.4-\min \{b, 0.5\}<0$, i.e., $b \leq 0.2$ and $b>0.4$, which give us an empty solution space in this case, or 2) $x>$ $(1-b) / 2$ when $b>0.2$ and $0.4-\min \{b, 0.5\}<0$, i.e., $b>0.4$, which is the same as the numerical results shown in Fig. 9a. Furthermore, we show the throughput capacity

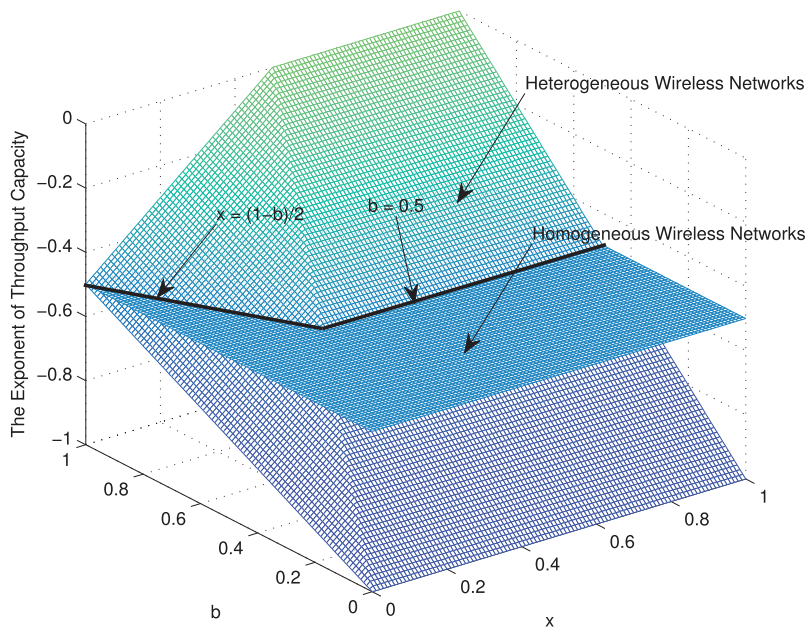

(a) $w=1$ and $d=1$ of heterogeneous networks when $b=0.5,0.6,0.7,0.8$, and 0.9 , respectively, in Fig. $9 \mathrm{~b}$. According to Corollary 2, since $b>-2 w+1$ in this example, the throughput capacity can only increase as the the bandwidth when $x<\min \{0.5 b+$ $0.1,-0.5 b+0.6\}$, i.e., $x<0.35$ when $b=0.5, x<0.3$ when $b=0.6, x<0.25$ when $b=0.7, x<0.2$ when $b=0.8$, and $x<0.15$ when $b=0.9$, respectively. Higher bandwidth than that will not enhance the capacity further because of the limitation of the number of destination nodes, which matches the numerical results in Fig. 9b well. A special case when $w=0.5$ and $d=1$, i.e., a network with square network area and symmetric traffic pattern, is shown in Fig. 10. We can see that heterogeneous wireless networks can achieve higher capacity than homogeneous wireless networks when $x>(1-b) / 2$ and $b>0.5$, which is consistent with Corollary 1 , and that the capacity of heterogeneous networks can increase as the bandwidth as long as

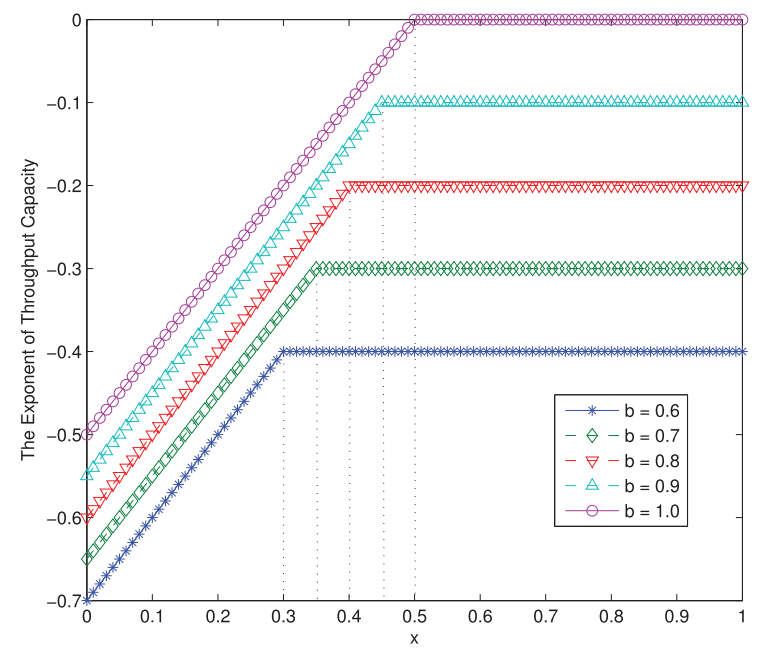

(b) $w=1, d=1$, and $b=0.6 \sim 1$

Fig. 10. Simulation results when $w=1$ and $d=1$. (a) The throughput capacity of homogeneous wireless networks and of heterogeneous wireless networks. (b) The throughput capacity of heterogeneous wireless networks when $b=0.6,0.7,0.8,0.9$, and 1.0 , respectively. 
$x<0.3$ when $b=0.6, x<0.35$ when $b=0.7, x<0.4$ when $b=0.8, x<0.45$ when $b=0.9$, and $x<0.5$ when $b=1.0$, which matches Corollary 2, i.e., $x<\min \{b / 2,1-b / 2\}=b / 2$.

\section{Conclusion}

As wireless technologies advance, there is no doubt that we will have more and more wireless devices surrounding us, which could have different capabilities and be used for different purposes. Thus, heterogeneity is indeed an inherent property of the future wireless networks. Unfortunately, it has not been embraced into capacity analysis so far. In this study, we investigate the throughput capacity of regular and random heterogeneous wireless networks, respectively, and find that many network factors such as the shape of the network area, the number of the destination nodes, the number of the helping nodes, and the bandwidth of the helping nodes, all have great impacts on the network capacity. We also find that by deploying wireless helping nodes into the network, heterogeneous wireless networks can provide much higher per-node throughput than traditional homogeneous wireless networks under certain conditions.

Recall that it has been shown in the literature that hybrid wireless networks can also achieve higher throughput than homogeneous networks. However, one assumption there is that the wired network connecting base stations has unlimited bandwidth. One natural question is thus: How much on earth should the bandwidth of the backbone network be in order to provide higher throughput? This question is of more interest in heterogeneous wireless networks where some powerful helping nodes (such as routers deployed in a city equipped with MIMO, UWB, cognitive radio, etc.) have much higher bandwidth than normal users (such as WiFi enabled PDAs, smart phones, etc.). We have addressed the question in this paper. The results show that if we rely on the helping nodes to relay all the packets, their bandwidth needs to be bounded by $\omega\left(n^{w}\right)$ when $w+b / 2-1 / 2 \leq 0$ and $w-\min \{b, d\}<0$, and by $\omega\left(n^{\frac{1-b}{2}}\right)$ when $w+b / 2-1 / 2>0$ and $w-\min \{b, d\}<0$, respectively. Also, notice that increased helping nodes' bandwidth does not necessarily always lead to increased network throughput. We find that the bandwidth of the helping nodes should be upper bounded by $\min \left\{n^{b}, n^{d}\right\}$ when $w+b / 2-1 / 2 \leq 0$, and by $\min \left\{n^{\frac{b}{2}-w+\frac{1}{2}}, n^{d-\frac{b}{2}-w+\frac{1}{2}}\right\}$ when $w+b / 2-1 / 2>0$, respectively. More bandwidth than that cannot increase the network throughput further.

\section{ACKNOWLEDGMENTS}

This work was partially supported by the US National Science Foundation under grants CNS-0721744, CNS0916391, CNS-1147813, CNS-1147851, and CNS-1149786. The work of Y. Fang was also partially supported by the National Natural Science Foundation of China under grant no. 61003300 and the 111 Project under B08038 with Xidian University, China. An earlier version of this paper was presented at INFOCOM 2010 [21].

\section{REFERENCES}

[1] A. Agarwal and P. Kumar, "Capacity Bounds for Ad Hoc and Hybrid Wireless Networks," ACM SIGCOMM, vol. 34, no. 3, pp. 71-81, July 2004.

[2] O. Arpacioglu and Z. Haas, "On the Scalability and Capacity of Wireless Networks with Omnidirectional Antennas," Proc. Third Int'l Symp. Information Processing in Sensor Networks (IPSN), Apr. 2004.

[3] A. Avudainayagam, Y. Fang, and W. Lou, "Dear: A Device and Energy Aware Routing Protocol for Heterogeneous Ad Hoc Networks," J. Parallel and Distributed Computing, vol. 63, no. 2, pp. 228-236, Feb. 2003.

[4] F. Baccelli and B. Blaszczyszyn, “On a Coverage Process Ranging from the Boolean Model to the Poisson Voronoi Tessellation, with Applications to Wireless Communications," Advances in Applied Probability, vol. 33, no. 2, 293-323, 2001.

[5] N. Bansal and Z. Liu, "Capacity, Delay, and Mobility in Wireless Ad-Hoc Networks," Proc. IEEE INFOCOM, Mar. 2003.

[6] C. Buraagohain, S. Suri, C. Toth, and Y. Zhou, "Improved Throughput Bounds for Interference-Aware Routing in Wireless Networks," Proc. Int'l Conf. Computing and Combinatorics (COCOON), July 2007.

[7] T. Cormen, C. Leiserson, R. Rivest, and C. Stein, Introduction to Algorithms, second ed. MIT, 2001.

[8] O. Dousse, F. Baccelli, and P. Thiran, "Impact of Interferences on Connectivity in Ad Hoc Networks," Proc. IEEE INFOCOM, Apr. 2003.

[9] O. Dousse, M. Franceschetti, and P. Thiran, "On the Throughput Scaling of Wireless Relay Networks," IEEE Trans. Information Theory, vol. 52, no. 6, 2756-2761, June 2006.

[10] E. Duarte-Melo, A. Josan, M. Liu, D. Neuhoff, and S. Pradhan, "The Effect of Node Density and Propagation Model on Throughput Scaling of Wireless Networks," Proc. IEEE Int'l Symp. Information Theory (ISIT), July 2006.

[11] M. Franceschetti, O. Dousse, D.N. Tse, and P. Thiran, "Closing the Gap in the Capacity of Wireless Networks via Percolation Theory," IEEE Trans. Information Theory, vol. 53, no. 3, pp. 10091018, Mar. 2007.

[12] L. Fu, Y. Qin, X. Wang, and X. Liu, "Converge-Cast with MIMO," Proc. IEEE INFOCOM, Apr. 2011.

[13] M. Grossglauser and D. Tse, "Mobility Increases the Capacity of Ad Hoc Wireless Networks," IEEE/ACM Trans. Networking, vol. 10, no. 4, pp. 477-486, Aug. 2002.

[14] P. Gupta and P. Kumar, "The Capacity of Wireless Networks," IEEE Trans. Information Theory, vol. 46, no. 2, 388-404, Mar. 2000.

[15] K. Jain, J. Padhye, V.N. Padmanabhan, and L. Qiu, "Impact of Interference on Multi-Hop Wireless Network Performance," Wireless Networks, vol. 1, pp. 471-487, July 2005.

[16] K. Kenthapadi and R. Panigrahy, "Balanced Allocation on Graphs," Proc. 17th Ann. ACM-SIAM Symp. Discrete Algorithm (SODA '06), 2006.

[17] D. Knuth, The Art of Computer Programming. Addison-Wesley, 1998.

[18] U. Kozat and L. Tassiulas, "Throughput Capacity of Random Ad Hoc Networks with Infrastructure Support," Proc. ACM MobiCom, June 2003.

[19] N. Li and J. Hou, "Topology Control in Heterogeneous Wireless Networks: Problems and Solutions," Proc. IEEE INFOCOM, Mar. 2004.

[20] P. Li and Y. Fang, "Impacts of Topology and Traffic Pattern on Capacity of Hybrid Wireless Networks," IEEE Trans. Mobile Computing, vol. 8, no. 12, pp. 1585-1595, Dec. 2009.

[21] P. Li and Y. Fang, "The Capacity of Heterogeneous Wireless Networks," Proc. IEEE INFOCOM, Mar. 2010.

[22] P. Li, Y. Fang, and J. Li, "Throughput, Delay, and Mobility in Wireless Ad Hoc Networks," Proc. IEEE INFOCOM, Mar. 2010.

[23] P. Li, X. Geng, and Y. Fang, "An Adaptive Power Controlled MAC Protocol for Wireless Ad Hoc Networks," IEEE Trans. Wireless Comm., vol. 8, no. 1, pp. 226-233, Jan. 2009.

[24] P. Li, X. Huang, and Y. Fang, "Capacity Scaling of Multihop Cellular Networks," Proc. IEEE INFOCOM, Apr. 2011.

[25] P. Li, Q. Shen, Y. Fang, and H. Zhang, "Power Controlled Network Protocols for Multi-Rate Ad Hoc Networks," IEEE Trans. Wireless Comm., vol. 8, no. 4, pp. 2142-2149, Apr. 2009.

[26] P. Li, C. Zhang, and Y. Fang, "The Capacity of Wireless Ad Hoc Networks Using Directional Antennas," IEEE Trans. Mobile Computing, vol. 10, no. 10, pp. 1374-1387, Oct. 2011. 
[27] P. Li, C. Zhang, and Y. Fang, "Asymptotic Connectivity in Wireless Ad Hoc Networks Using Directional Antenna," IEEE/ ACM Trans. Networking, vol. 17, no. 4, pp. 1106-1117, Aug. 2009.

[28] X. Lin, G. Sharma, R. Mazumdar, and N. Shroff, "Degenerate Delay-Capacity Tradeoffs in Ad-Hoc Networks with Brownian Mobility," IEEE/ACM Trans. Networking, vol. 14, pp. 2777-2784, June 2006

[29] B. Liu, Z. Liu, and D. Towsley, "On the Capacity of Hybrid Wireless Networks," Proc. IEEE INFOCOM, Mar. 2003.

[30] B. Liu, P. Thiran, and D. Towsley, "Capacity of a Wireless Ad Hoc Network with Infrastructure," Proc. ACM MobiHoc, Sept. 2007.

[31] W. Liu, Y. Zhang, and Y. Fang, "Conserving Energy in Heterogeneous Mobile Ad Hoc Networks," Proc. IEEE Military Comm. Conf. (Milcom'05), Oct. 2005.

[32] W. Liu, Y. Zhang, W. Lou, and Y. Fang, "DELAR: Device/Energy/ Load Aware Relaying in Heterogeneous Wireless Ad Hoc Networks," Proc. IEEE Military Comm. Conf. (Milcom '04), Nov. 2004

[33] W. Liu, Y. Zhang, K. Lu, and Y. Fang, "Energy Conservation through Resource-Aware Movement in Heterogeneous Mobile Ad Hoc Networks," J. Combinatorial Optimization, vol. 11, no. 1, pp. 7-20, Feb. 2006.

[34] J. Mammen and D. Shah, "Throughput and Delay in Random Wireless Networks with Restricted Mobility," IEEE Trans. Information Theory, vol. 53, no. 3, pp. 1108-1116, Mar. 2007.

[35] M. McGarry, M. Reisslein, and V. Syrotiuk, "Access Control in Heterogeneous Multichannel Wireless Networks," Proc. First Int'l Conf. Integrated Internet Ad hoc and Sensor Networks (InterSense '06), May 2006.

[36] A. Ozgur, O. Leveque, and D. Tse, "How Does the Information Capacity of Ad Hoc Networks Scale?" Proc. 44th Ann. Allerton Conf. Comm., Control and Computing, Sept. 2006.

[37] C. Peraki and S. Servetto, "On the Maximum Stable Throughput Problems in Random Networks with Directional Antennas," Proc. ACM MobiHoc, June 2003.

[38] T. Rappaport, Wireless Communications: Principles and Practice, second ed. Prentice-Hall PTR, 2002.

[39] R. Sollacher, M. Greiner, and I. Glauche, "Impact of Interference on the Wireless Ad-Hoc Networks Capacity and Topology," Wireless Networks, vol. 12, pp. 53-61, Feb. 2006.

[40] S. Toumpis, "Capacity Bounds for Three Classes of Wireless Networks," Proc. ACM MobiHoc, May 2004.

[41] X. Wang, Y. Bei, Q. Peng, and L. Fu, "Speed Improves DelayCapacity Tradeoff in Motioncast," IEEE Trans. Parallel and Distributed Systems, vol. 1, no. 99, pp. 1-13, 2010.

[42] X. Wang, W. Huang, S. Wang, J. Zhang, and C. Hu, "Delay and Capacity Tradeoff Analysis for Motioncast," IEEE Trans. Networking, vol. 19, no. 5, pp. 1354-1367, Oct. 2011.

[43] Wikipedia, "Utah," http://en.wikipedia.org/wiki/Utah, 2012.

[44] E. Wu and Y. Huang, "Dynamic Adaptive Routing for a Heterogeneous Wireless Network," Mobile Networks and Applications, vol. 9, no. 3, pp. 219-233, June 2004.

[45] K. Yang, Y. Wu, and H.-H. Chen, "QoS-Aware Routing in Emerging Heterogeneous Wireless Networks," IEEE Comm. Magazine, vol. 45, no. 2, pp. 74-80, Feb. 2007.

[46] A. Zemlianov and G. Veciana, "Capacity of Ad Hoc Wireless Networks with Infrastructure Support," IEEE J. Selected Areas in Comm., vol. 23, no. 3, pp. 657-667, Mar. 2005.

[47] G. Zhang, Y. Xu, X. Wang, and M. Guizani, "Capacity of Hybrid Wireless Networks with Directional Antenna and Delay Constraint," IEEE Trans. Comm., vol. 58, no. 7, pp. 2097-2106, July 2010.

[48] S. Zhao and D. Raychaudhuri, "On the Scalability of Hierarchical Hybrid Wireless Networks," Proc. IEEE 40th Ann. Conf. Information Sciences and Systems (CISS), Mar. 2006.

[49] H. Zhou, C. Yeh, and H. Mouftah, "Access Control in Heterogeneous Multichannel Wireless Networks," Proc. IEEE 60th Vehicular Technology Conf., Sept. 2004.

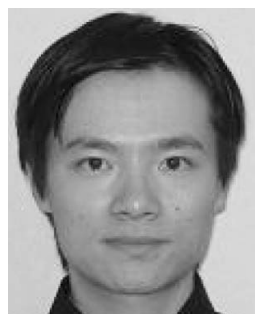

Pan $\mathbf{L i}$ received the $\mathrm{BE}$ degree in electrical engineering from the Huazhong University of Science and Technology, Wuhan, China, in 2005, and the PhD degree in electrical and computer engineering from the University of Florida, Gainesville, in 2009, respectively. Currently, he is working as an assistant professor in the Department of Electrical and Computer Engineering, Mississippi State University. His research interests include capacity and connectivity investigation, cross-layer optimization and design, and security and privacy in wireless networks, complex networks, cyber-physical systems, mobile computing, and cloud computing. He serves as an editor for the IEEE Journal on Selected Areas in Communications-Cognitive Radio Series and IEEE Communications Surveys and Tutorials, as a feature editor for IEEE Wireless Communications, and as a guest editor for the IEEE Wireless Communications special issue on user cooperation in wireless networks. He received the US National Science Foundation CAREER Award in 2012 and is a member of the IEEE and the ACM.

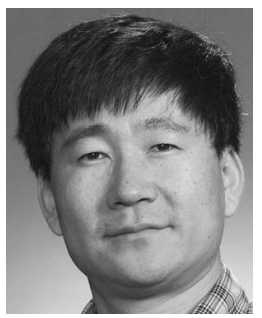

Yuguang Fang received the $\mathrm{PhD}$ degree in systems engineering from Case Western Reserve University in January 1994 and the PhD degree in electrical engineering from Boston University in May 1997. He was an assistant professor in the Department of Electrical and Computer Engineering at the New Jersey Institute of Technology from July 1998 to May 2000. He then joined the Department of Electrical and Computer Engineering at the University of Florida (UF) in May 2000 as an assistant professor, got an early promotion to associate professor with tenure in August 2003, and to full professor in August 2005. He held a University of Florida Research Foundation (UFRF) Professorship from 2006 to 2009, a Changjiang Scholar Chair Professorship with Xidian University, Xi'an, China, from 2008 to 2011, and a Guest Chair Professorship with Tsinghua University, China, from 2009 to 2012. He has published more than 300 papers in refereed professional journals and conferences. Dr. Fang received the US National Science Foundation Faculty Early Career Award in 2001, the US Office of Naval Research Young Investigator Award in 2002, Best Paper Awards from IEEE GlobeCom 2011 and the IEEE International Conference on Network Protocols (ICNP) 2006, and the IEEE TCGN Best Paper Award from the IEEE High-Speed Networks Symposium, IEEE GlobeCom 2002. He also received a 2010-2011 UF Doctoral Dissertation Advisor/Mentoring Award, a 2011 Florida Blue Key/UF Homecoming Distinguished Faculty Award, and the 2009 UF College of Engineering Faculty Mentoring Award. Dr. Fang is also active in professional activities. He served as the editor-in-chief for IEEE Wireless Communications (2009-2012) and serves/served on several editorial boards of technical journals including the IEEE Transactions on Mobile Computing (2003-2008, 2011-present), IEEE Network (2012present), the IEEE Transactions on Communications (2000-2011), the IEEE Transactions on Wireless Communications (2002-2009), the IEEE Journal on Selected Areas in Communications (1999-2001), the IEEE Wireless Communications Magazine (2003-2009), and ACM Wireless Networks (2001-present). He served on the steering committee for the IEEE Transactions on Mobile Computing (2008-2010). He actively participates in professional conference organizations such as serving as the technical program cochair for IEEE INOFOCOM 2014, steering committee cochair for QShine (2004-2008), technical program vice-chair for IEEE INFOCOM 2005, technical program area chair for IEEE INFOCOM (2009-2013), technical program symposium cochair for IEEE GlobeCom 2004, and a member of the technical program committee for IEEE INFOCOM $(1998,2000,2003-2008)$. He is a fellow of the IEEE and a member of ACM.

For more information on this or any other computing topic, please visit our Digital Library at www.computer.org/publications/dlib. 